\title{
ELSEVIER_MINE_4780
}

\section{Application of near infrared sensors to preconcentration of hydrothermally-formed copper ore}

Shekwonyadu lyakwaria,*

si233@exeter.ac.uk

Hylke J. Glass ${ }^{a}$

Gavyn K. Rollinson ${ }^{\mathrm{a}}$

Przemyslaw B. Kowalczuk

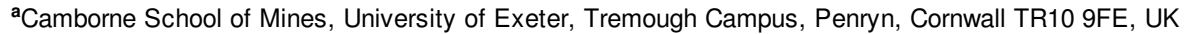

${ }^{b}$ Wroclaw University of Technology, Division of Mineral Processing, WybrzezeWyspianskiego 27, 50-370 Wroclaw, Poland

*Corresponding author.

\section{Abstract}

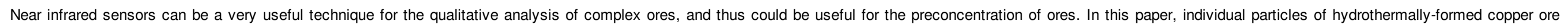

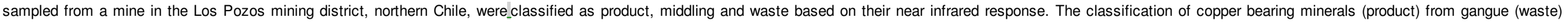

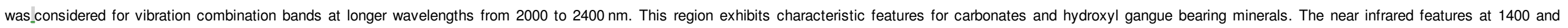
$1900 \mathrm{~nm}$ werenot considered favourable for classification and subsequent discrimination because they can be influenced by moisture and other environmental factors and are easily suppressed by iron-rich minerals.

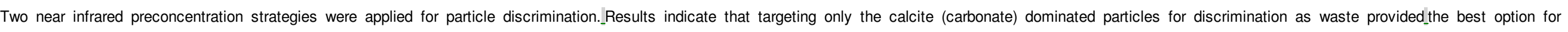

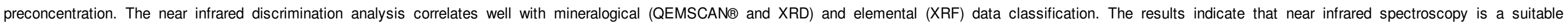
preconcentration method for supergene copper ore.

Keywords: Preconcentration; Near infrared; QEMSCAN®; XRD; Copper

\section{Introduction}

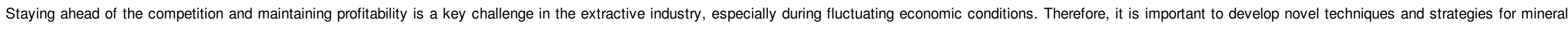

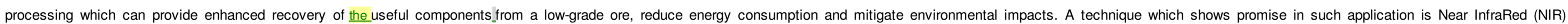
spectroscopy (Gaydon et al., 2009; Curtis, 2012; lyakwari et al., 2013; Dalm et al., 2014; lyakwari and Glass, 2015).

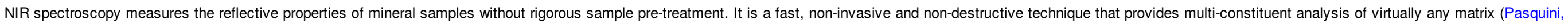

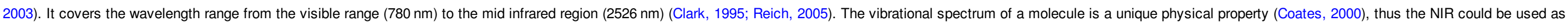

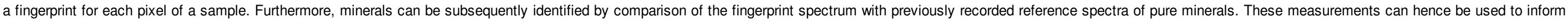
more generic mineral identification and classification methods (lyakwari et al., 2013).

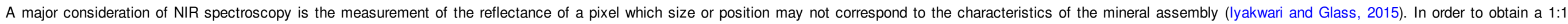

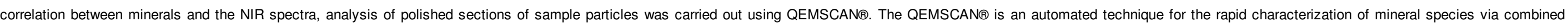

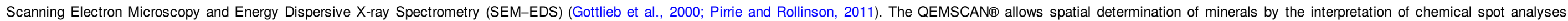
(Anderson et al., 2014). 


\section{ELSEVIER_MINE_4780}

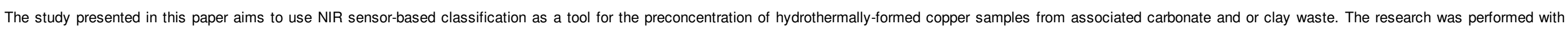

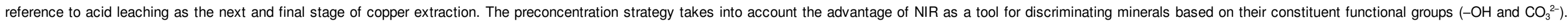
Also no two minerals display identical absorption features at the same wavelength positions within the NIR region, even if they contain the same functional groups_(Povarennykh, 1978; Iyakwari and Glass, 2014).

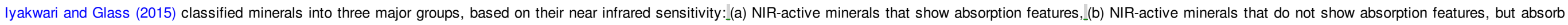

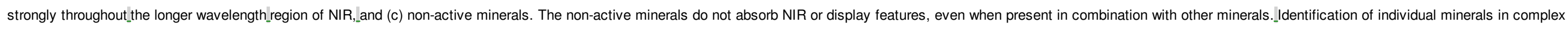

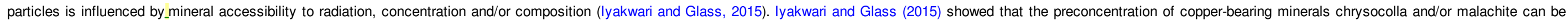

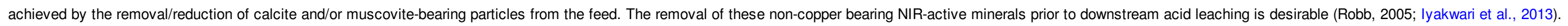

\section{Sample characterization and methods}

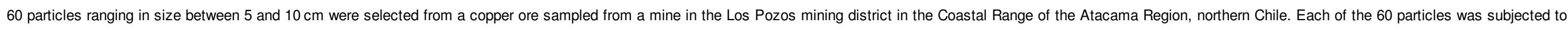
the characterization process outlined in Fig. 1.

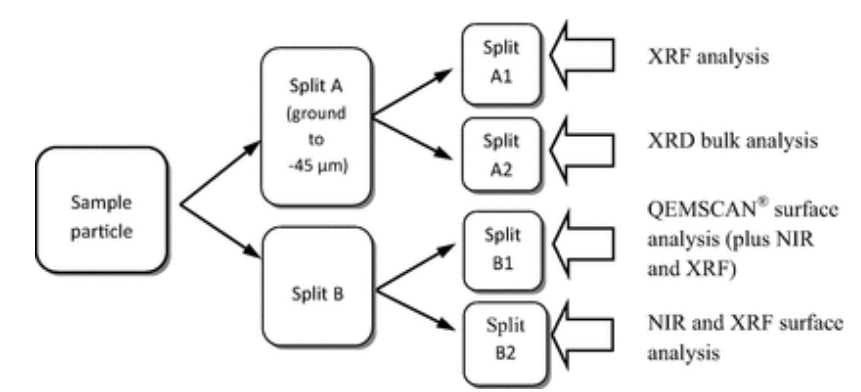

Fig. 1 Subdivision of sample particles for characterization.

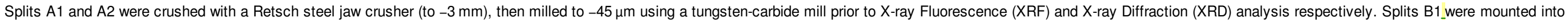

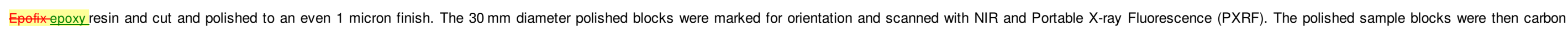

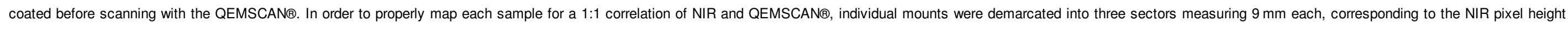

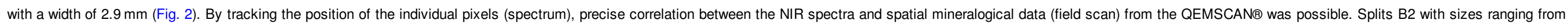
2 to $10 \mathrm{~cm}$ were scanned with the NIR and PXRF without any sample preparation, as the samples were dry and dust free.

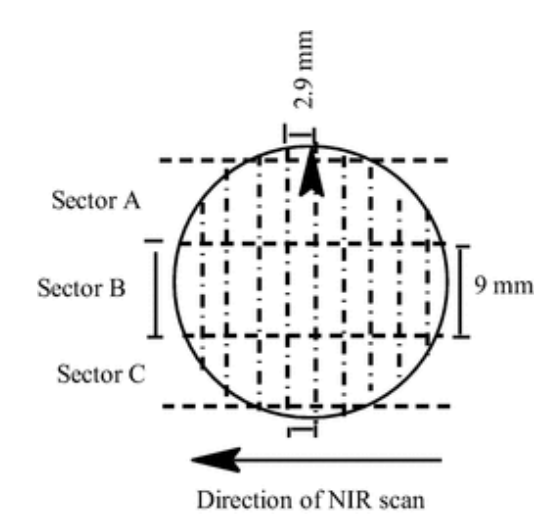

Fig. 2 Split B1 samples (in $30 \mathrm{~mm}$ diameter polished blocks) marked for directional scanning and spectral/mineral mapping.

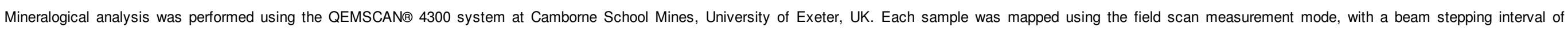




\section{ELSEVIER_MINE_4780}

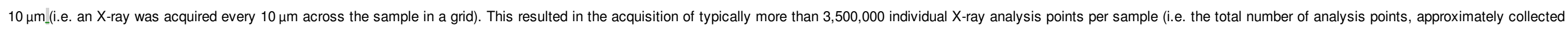

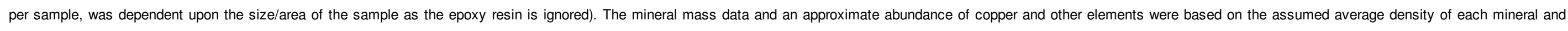

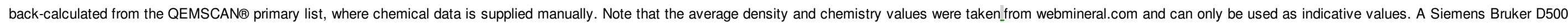

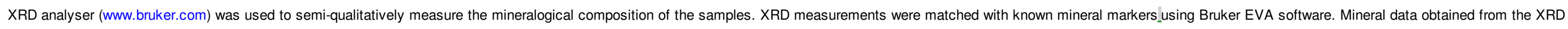
analysis were compared with the QEMSCAN® data.

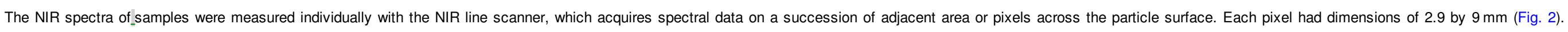
Details on NIR background, procedure of NIR data acquisition, instrumentation and data pre-treatment are described in lyakwari et al. (2013) and lyakwari and Glass (2015).

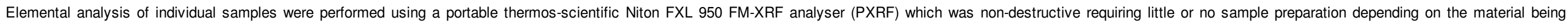
analysed. The PXRF analyser employs the Energy Dispersive X-ray Spectrometry (EDS) method (www.nitonuk.co.uk).

All analyses (NIR, QEMSCAN®, XRD and PXRF) were performed at the Camborne School of Mines (CSM) laboratory.

\section{Results}

\subsection{Ore mineralogy}

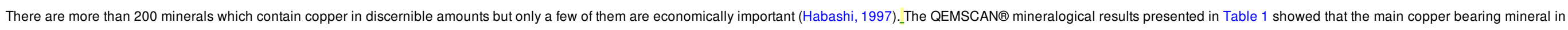

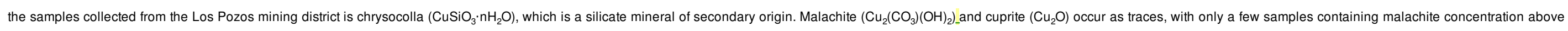
$1 \mathrm{wt} \%$.

Table 1 Modal mineralogy of the samples (mass $\%$ ) measured by QEMSCAN®,$<0.01 \%=$ below set detection limit.

\begin{tabular}{|c|c|c|c|c|c|c|c|c|c|c|c|c|c|c|c|c|c|}
\hline \multirow[t]{4}{*}{ Sample ID } & \multicolumn{10}{|c|}{ Silicates } & \multirow{2}{*}{\multicolumn{2}{|c|}{$\begin{array}{l}\text { Oxides } \\
\qquad \text { Cu-bearing }\end{array}$}} & \multicolumn{2}{|c|}{ Sarbonates } & Phosphates & \multirow[t]{4}{*}{ Others (trace phases) } & \multirow[t]{4}{*}{ Total } \\
\hline & Cu-bearing & \multicolumn{9}{|c|}{ Non-Cu-bearing } & & & \multicolumn{3}{|c|}{ Non-Cu-bearing } & & \\
\hline & \multicolumn{6}{|c|}{ NIR-active } & \multicolumn{3}{|c|}{ Non-active } & \multirow{2}{*}{$\begin{array}{l}\text { NIR-active } \\
\text { Hematite }\end{array}$} & \multirow{2}{*}{$\begin{array}{c}\text { Non-active } \\
\text { Cuprite }\end{array}$} & \multicolumn{4}{|c|}{ NIR-active } & & \\
\hline & Chrysocolla & Muscovite & Kaolinite & Biotite & Chlorite & Tourmaline & Quartz & K-feldspar & Plag-feldspar & & & Malachite & Calcite & Ankerite & Apatite & & \\
\hline 1 & 8.33 & 5.95 & 0.01 & 15.54 & 8.87 & 0.39 & 17.69 & 25.54 & 0.07 & 16.63 & 0.01 & $<0.01$ & 0.01 & 0.02 & 0.18 & 0.75 & 100 \\
\hline 2 & 19.05 & 3.79 & 0.01 & 8.32 & 6.68 & 0.3 & 14.96 & 26.21 & 0.03 & 19.9 & 0.02 & $<0.01$ & 0.01 & 0.04 & 0.27 & 0.41 & 100 \\
\hline 3 & 21.11 & 2.96 & 0.05 & 4.53 & 3.08 & 0.04 & 18.16 & 17.87 & 0.02 & 31.77 & 0.01 & 0.31 & $<0.01$ & $<0.01$ & $<0.01$ & 0.10 & 100 \\
\hline 4 & 5.14 & 3.28 & 0.68 & 7.30 & 5.13 & 0.72 & 6.84 & 15.50 & 0.06 & 55.32 & 0.01 & $<0.01$ & $<0.01$ & $<0.01$ & $<0.01$ & 0.02 & 100 \\
\hline 5 & 3.29 & 5.34 & $<0.01$ & 8.38 & 24.87 & 0.55 & 37.88 & 13.11 & 0.17 & 3.72 & 0.01 & 1.00 & 0.02 & 0.05 & 0.73 & 0.90 & 100 \\
\hline 6 & 1.09 & 1.00 & $<0.01$ & 15.32 & 6.64 & 0.13 & 9.86 & 32.42 & 1.16 & 10.37 & 0.04 & 0.01 & 17.17 & 2.88 & 0.13 & 1.78 & 100 \\
\hline 7 & 0.15 & 1.55 & $<0.01$ & 13.01 & 8.74 & 0.48 & 7.46 & 38.83 & 0.03 & 29.03 & $<0.01$ & $<0.01$ & 0.01 & 0.06 & 0.33 & 0.33 & 100 \\
\hline 8 & 5.58 & 0.22 & $<0.01$ & 5.96 & 15.76 & 0.10 & 4.91 & 38.01 & $<0.01$ & 28.47 & 0.01 & $<0.01$ & 0.01 & 0.02 & 0.16 & 0.79 & 100 \\
\hline 9 & 3.98 & 0.26 & $<0.01$ & 6.72 & 14.12 & 1.49 & 18.49 & 4.45 & 0.41 & 11.66 & 0.15 & 1.38 & 31.61 & 4.06 & 0.31 & 0.90 & 100 \\
\hline 10 & 7.78 & 1.62 & $<0.01$ & 5.86 & 4.47 & 0.13 & 15.13 & 1.32 & 0.01 & 62.04 & 0.03 & 1.55 & $<0.01$ & 0.01 & 0.01 & 0.03 & 100 \\
\hline 11 & 9.31 & 2.67 & 0.33 & 5.46 & 4.66 & 0.18 & 13.13 & 20.65 & 0.08 & 43.17 & $<0.01$ & $<0.01$ & $<0.01$ & $<0.01$ & $<0.01$ & 0.36 & 100 \\
\hline 12 & 6.92 & 3.55 & 0.43 & 4.90 & 3.47 & 0.27 & 11.19 & 19.44 & 0.05 & 49.74 & $<0.01$ & $<0.01$ & $<0.01$ & $<0.01$ & $<0.01$ & 0.04 & 100 \\
\hline
\end{tabular}




\section{ELSEVIER_MINE_4780}

\begin{tabular}{|c|c|c|c|c|c|c|c|c|c|c|c|c|c|c|c|c|c|}
\hline 13 & $<0.01$ & 2.31 & 0.02 & 3.33 & 2.99 & 0.06 & 18.37 & 11.74 & 0.30 & 53.44 & $<0.01$ & $<0.01$ & 5.08 & 2.03 & $<0.01$ & 0.31 & 100 \\
\hline 14 & 1.19 & 3.73 & 1.16 & 6.71 & 13.43 & 2.40 & 16.14 & 11.15 & 0.82 & 43.06 & $<0.01$ & $<0.01$ & 0.10 & 0.05 & $<0.01$ & 0.07 & 100 \\
\hline 15 & $<0.01$ & 8.38 & 0.04 & 22.89 & 10.85 & 0.07 & 7.69 & 33.62 & 0.21 & 6.98 & $<0.01$ & $<0.01$ & 3.77 & 2.64 & 0.03 & 2.84 & 100 \\
\hline 16 & 0.40 & 0.12 & $<0.01$ & 9.42 & 15.16 & 0.06 & 38.98 & 34.25 & $<0.01$ & 0.63 & $<0.01$ & 0.01 & 0.01 & $<0.01$ & 0.29 & 0.69 & 100 \\
\hline 17 & 3.46 & 0.02 & $<0.01$ & 0.51 & 25.61 & 0.11 & 64.6 & 0.76 & 0.03 & 3.41 & 0.02 & 0.39 & 0.02 & $<0.01$ & 0.17 & 0.90 & 100 \\
\hline 18 & 0.27 & 17.73 & $<0.01$ & 4.59 & 2.45 & 0.03 & 41.9 & 30.3 & 0.06 & 0.22 & $<0.01$ & $<0.01$ & 1.08 & 0.64 & 0.02 & 0.71 & 100 \\
\hline 19 & 0.45 & 9.90 & $<0.01$ & 17.7 & 5.37 & 0.30 & 20.54 & 16.95 & 1.16 & 7.13 & $<0.01$ & $<0.01$ & 16.28 & 2.45 & 0.25 & 1.53 & 100 \\
\hline 20 & 0.81 & 1.67 & $<0.01$ & 10.1 & 36.47 & 0.36 & 38.26 & 10.07 & 0.05 & 0.98 & 0.01 & 0.14 & 0.03 & $<0.01$ & 0.24 & 0.79 & 100 \\
\hline 21 & 0.07 & 18.82 & 3.11 & 4.77 & 4.20 & 0.25 & 31.00 & 16.03 & 2.26 & 19.18 & $<0.01$ & $<0.01$ & $<0.01$ & $<0.01$ & $<0.01$ & 0.31 & 100 \\
\hline 22 & 0.08 & 0.25 & $<0.01$ & 6.99 & 11.25 & 0.09 & 46.08 & 33.64 & 0.01 & 0.12 & $<0.01$ & $<0.01$ & 0.01 & 0.01 & 0.66 & 0.82 & 100 \\
\hline 23 & 0.05 & 0.05 & $<0.01$ & 1.78 & 33.81 & 1.13 & 57.69 & 3.13 & 0.20 & 0.89 & $<0.01$ & $<0.01$ & 0.03 & 0.01 & 0.21 & 1.02 & 100 \\
\hline 24 & 1.23 & 21.55 & $<0.01$ & 3.46 & 1.33 & 0.05 & 62.89 & 5.73 & 0.03 & 2.92 & 0.01 & 0.66 & 0.01 & 0.01 & 0.07 & 0.05 & 100 \\
\hline 25 & 0.36 & 0.10 & $<0.01$ & 3.36 & 17.66 & 0.47 & 51.90 & 4.50 & 0.30 & 0.51 & $<0.01$ & $<0.01$ & 18.81 & 0.45 & 0.38 & 1.21 & 100 \\
\hline 26 & 3.26 & 4.14 & $<0.01$ & 8.47 & 26.35 & 0.40 & 36.34 & 14.00 & 0.15 & 4.13 & 0.01 & 1.20 & 0.02 & 0.04 & 0.46 & 1.04 & 100 \\
\hline 27 & $<0.01$ & 10.55 & 0.03 & 5.08 & 2.22 & 0.44 & 25.81 & 18.98 & 3.80 & 0.18 & $<0.01$ & $<0.01$ & 28.89 & 0.27 & 0.25 & 3.51 & 100 \\
\hline 28 & 1.64 & $<0.01$ & 0.01 & 0.84 & 35.08 & 0.80 & 57.24 & 0.10 & 0.50 & 1.03 & 0.01 & 0.62 & 0.05 & 0.01 & 0.96 & 1.10 & 100 \\
\hline 29 & 0.21 & 14.84 & 0.02 & 3.06 & 1.27 & 0.51 & 20.72 & 11.64 & 3.51 & 1.22 & $<0.01$ & $<0.01$ & 38.82 & 0.81 & 0.27 & 3.10 & 100 \\
\hline 30 & 0.08 & 0.14 & 0.02 & 3.54 & 35.29 & 2.49 & 50.49 & 4.38 & 1.02 & 1.16 & $<0.01$ & $<0.01$ & 0.02 & 0.01 & 0.39 & 0.96 & 100 \\
\hline 31 & 0.27 & 1.62 & $<0.01$ & 5.93 & 45.02 & 0.36 & 30.31 & 10.25 & 0.12 & 4.02 & $<0.01$ & $<0.01$ & 0.05 & 0.03 & 0.86 & 1.16 & 100 \\
\hline 32 & 2.05 & 0.22 & $<0.01$ & 1.16 & 34.74 & 0.10 & 49.57 & 2.43 & 0.13 & 0.74 & 0.02 & 0.91 & 0.35 & 0.13 & 4.99 & 2.47 & 100 \\
\hline 33 & $<0.01$ & 11.26 & 0.14 & 16.28 & 5.24 & 0.17 & 10.64 & 40.11 & 0.98 & 3.05 & $<0.01$ & $<0.01$ & 8.01 & 2.13 & 0.05 & 1.94 & 100 \\
\hline 34 & 0.22 & 0.04 & $<0.01$ & 1.69 & 36.54 & 0.25 & 56.91 & 2.32 & 0.05 & 0.26 & $<0.01$ & $<0.01$ & 0.05 & 0.01 & 0.35 & 1.31 & 100 \\
\hline 35 & 2.98 & 1.65 & $<0.01$ & 7.24 & 19.10 & 0.06 & 21.38 & 10.43 & 0.26 & 3.08 & 0.23 & 0.85 & 27.02 & 4.10 & 0.53 & 1.10 & 100 \\
\hline 36 & 0.02 & 0.54 & $<0.01$ & 12.15 & 16.30 & 0.06 & 30.12 & 34.5 & 0.07 & 0.73 & $<0.01$ & $<0.01$ & 3.78 & 0.12 & 0.18 & 1.43 & 100 \\
\hline 37 & 0.82 & 0.08 & $<0.01$ & 15.69 & 43.83 & 0.02 & 0.40 & 35.92 & $<0.01$ & 1.72 & $<0.01$ & $<0.01$ & 0.01 & $<0.01$ & 0.21 & 1.31 & 100 \\
\hline 38 & 0.18 & 1.41 & $<0.01$ & 40.82 & 7.89 & 0.17 & 21.90 & 22.92 & 0.05 & 3.89 & $<0.01$ & $<0.01$ & 0.03 & 0.02 & 0.42 & 0.31 & 100 \\
\hline 39 & 0.98 & 0.11 & $<0.01$ & 10.24 & 45.84 & 0.02 & 7.89 & 32.78 & 0.01 & 0.27 & $<0.01$ & $<0.01$ & 0.02 & 0.01 & 0.53 & 1.33 & 100 \\
\hline 40 & 0.89 & 0.06 & $<0.01$ & 1.59 & 19.61 & 0.94 & 72.21 & 3.8 & 0.05 & 0.02 & $<0.01$ & $<0.01$ & 0.01 & $<0.01$ & 0.06 & 0.76 & 100 \\
\hline 41 & $<0.01$ & $<0.01$ & 0.01 & 2.95 & 23.19 & 9.41 & 29.54 & $<0.01$ & 3.99 & 3.48 & $<0.01$ & $<0.01$ & 13.1 & 6.04 & 0.17 & 8.12 & 100 \\
\hline 42 & 0.27 & 6.12 & $<0.01$ & 11.81 & 4.56 & 0.34 & 20.31 & 11.18 & 1.79 & 0.43 & $<0.01$ & $<0.01$ & 40.52 & 0.56 & 0.26 & 1.86 & 100 \\
\hline 43 & 0.04 & 4.96 & 0.10 & 8.37 & 6.28 & 1.58 & 30.74 & 10.56 & 9.08 & 1.12 & $<0.01$ & $<0.01$ & 18.45 & 0.41 & 0.32 & 8.00 & 100 \\
\hline 44 & 0.15 & 0.21 & $<0.01$ & 3.14 & 40.72 & 0.06 & 41.10 & 1.71 & 0.05 & 0.35 & $<0.01$ & $<0.01$ & 10.11 & 0.25 & 0.65 & 1.50 & 100 \\
\hline 45 & 0.54 & 0.16 & $<0.01$ & 7.00 & 15.58 & 0.03 & 3.19 & 60.96 & 0.07 & 2.38 & $<0.01$ & $<0.01$ & 8.55 & 0.38 & 0.22 & 0.94 & 100 \\
\hline
\end{tabular}


ELSEVIER_MINE_4780

\begin{tabular}{|c|c|c|c|c|c|c|c|c|c|}
\hline 7 & + & + & & & & & + & & \\
\hline 8 & + & + & & + & & & + & & + \\
\hline 9 & + & & & + & + & & + & & + \\
\hline 10 & + & + & + & & & & & & + \\
\hline 11 & + & + & & & & & + & & + \\
\hline 13 & + & + & & & & & & & \\
\hline 14 & + & + & + & & & & + & & \\
\hline 15 & + & & & & & + & & + & \\
\hline 16 & + & & & + & & & & + & \\
\hline 17 & + & & & + & & & & & \\
\hline 18 & + & & + & + & & & + & & \\
\hline 19 & + & + & & + & + & + & & & \\
\hline 20 & + & & + & + & & & & & \\
\hline 21 & + & + & + & & & & & + & \\
\hline 22 & + & & & & & & & + & \\
\hline 24 & + & + & + & & & & & & + \\
\hline 26 & + & & + & + & & & + & & \\
\hline 27 & + & & + & & + & & & & \\
\hline 28 & + & & & + & & & & & \\
\hline 30 & + & & + & + & & & + & & \\
\hline 31 & + & & + & + & & & + & & \\
\hline 32 & + & & & + & & & & & \\
\hline 34 & + & & & + & & & & & \\
\hline 35 & + & & & + & + & & & & + \\
\hline 36 & + & & & + & & & & + & \\
\hline 37 & + & & & + & + & & & + & \\
\hline 38 & + & & & + & & + & & & \\
\hline 39 & + & & & + & & & + & & \\
\hline 40 & + & & & + & & & + & & \\
\hline 42 & + & & + & + & + & & + & & \\
\hline 43 & + & & + & & + & & & & \\
\hline 45 & + & & & + & + & & & + & \\
\hline 46 & + & & + & & + & & & & \\
\hline
\end{tabular}
列 (
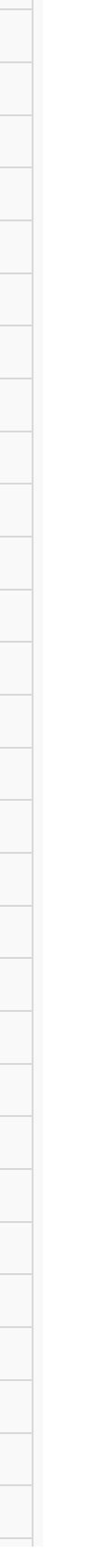

80




\section{ELSEVIER_MINE_4780}

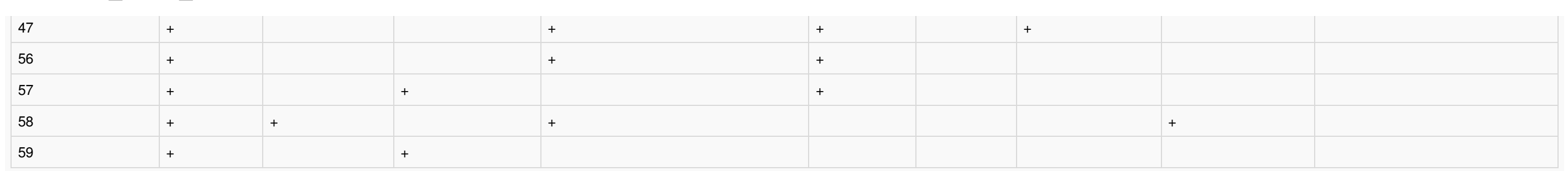

Both QEMSCAN® and XRD results showed that in all investigated samples, there were only three major mineral groups: oxides, carbonates and silicates dominated the ore, with phosphate (apatite) occurring as traces.

\subsection{Influence of resin used on NIR signal}

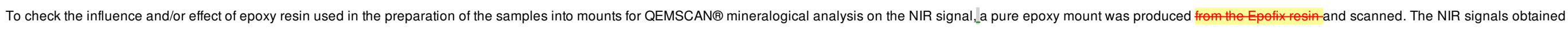
were treated with the same method as the rock samples and presented in Fig. 3.

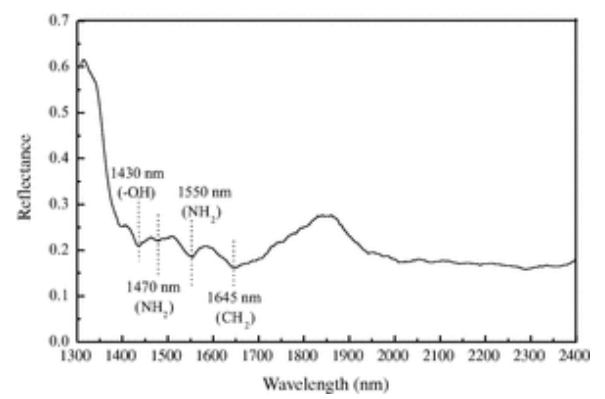

Fig. 3 NIR spectra of Epofix (epoxy resin).

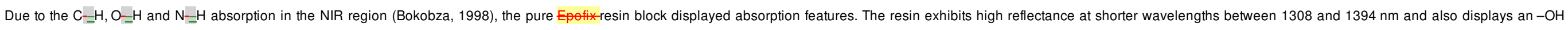

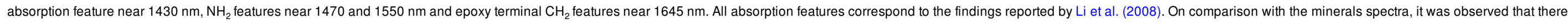

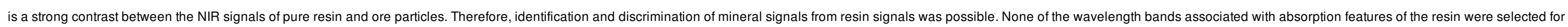
preconcentration of the investigated ore.

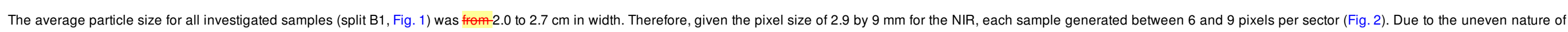

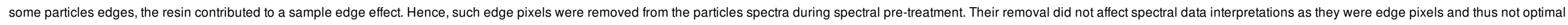
for analysis.

\subsection{NIR-active functional groups}

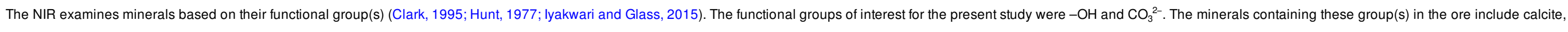

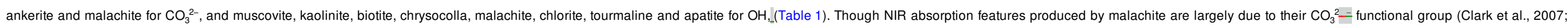

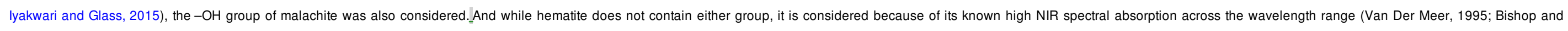
Dummel, 1996; lyakwari and Glass, 2015). It is important to note that functional groups like $\mathrm{CO}_{3}{ }^{2}=$ tend to consume acid while groups like -_OH, especially the clays, adsorb acid (Gupta, 2003; Curtis, 2012).

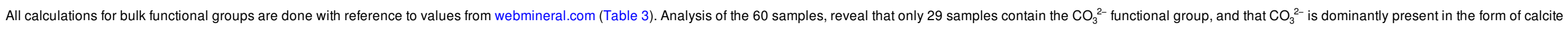

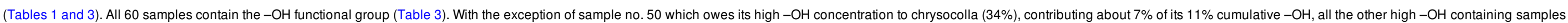
are dominantly as a result of their chlorite concentration (Table 3). Hematite was detected in all samples, with only 16 samples showing hematite below 1 wt\% (Table 1). 


\section{ELSEVIER_MINE_4780}

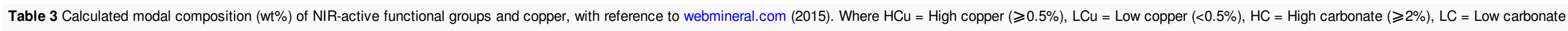
$(<2 \%), \mathrm{HOH}=$ High Hydroxyl $(\geqslant 5 \%), \mathrm{LOH}=$ Low Hydroxyl $(<5 \%), \mathrm{HH}=$ High Hematite $(\geqslant 10 \%)$, and LH = Low Hematite $(<10 \%)$.

\begin{tabular}{|c|c|c|c|c|c|c|c|c|c|c|c|c|c|c|c|c|c|}
\hline \multirow{3}{*}{$\begin{array}{l}\text { Sample } \\
\text { ID }\end{array}$} & \multicolumn{8}{|c|}{ Hydroxyls } & \multicolumn{3}{|c|}{ Carbonates } & \multirow{3}{*}{$\begin{array}{c}\text { Cumulative } \\
-\mathrm{OH}\end{array}$} & \multirow{3}{*}{$\begin{array}{l}\text { Cumulative } \\
\mathrm{CO}_{3}{ }^{2-}\end{array}$} & \multirow[t]{3}{*}{ Copper } & \multicolumn{3}{|c|}{ Classification } \\
\hline & \multirow[t]{2}{*}{$\begin{array}{c}\text { Muscovite } \\
8.53 \%(-\mathrm{OH})\end{array}$} & \multirow{2}{*}{$\begin{array}{l}\text { Biotite } \\
7.84 \% \\
(-\mathrm{OH})\end{array}$} & \multirow{2}{*}{$\begin{array}{c}\text { Kaolinite } \\
26.34 \% \\
(-\mathrm{OH})\end{array}$} & \multirow{2}{*}{$\begin{array}{l}\text { Chlorite } \\
22.85 \% \\
(-\mathrm{OH})\end{array}$} & \multirow[t]{2}{*}{$\begin{array}{l}\text { Tourmaline } \\
6.61 \%(-\mathrm{OH})\end{array}$} & \multirow[t]{2}{*}{$\begin{array}{l}\text { Chrysocolla } \\
20.71 \%(-\mathrm{OH})\end{array}$} & \multirow{2}{*}{$\begin{array}{l}\text { Apatite } \\
3.34 \% \\
(-\mathrm{OH})\end{array}$} & \multicolumn{2}{|c|}{$\begin{array}{c}\text { Malachite } 15.38 \%(-\mathrm{OH}) \\
\text { and } 27.13 \%\left(\mathrm{CO}_{3}{ }^{2-}\right)\end{array}$} & \multirow{2}{*}{$\begin{array}{c}\text { Calcite } \\
59.95 \% \\
\left(\mathrm{CO}_{3}{ }^{2-}\right)\end{array}$} & \multirow{2}{*}{$\begin{array}{c}\text { Ankerite } \\
58.14 \% \\
\left(\mathrm{CO}_{3}{ }^{2-}\right)\end{array}$} & & & & \multirow{2}{*}{$\begin{array}{c}\text { Copper } \\
- \\
\mathrm{CO}_{3}^{2-}\end{array}$} & \multirow[t]{2}{*}{$\begin{array}{l}\text { Copper } \\
--\mathrm{OH}\end{array}$} & \multirow{2}{*}{$\begin{array}{l}\text { Copper } \\
- \\
\text { Hematite }\end{array}$} \\
\hline & & & & & & & & $-\mathrm{OH}$ & $\mathrm{CO}_{3}^{2-}$ & & & & & & & & \\
\hline 1 & 0.51 & 1.22 & 0.00 & 2.03 & 0.03 & 1.73 & 0.01 & 0.00 & 0.00 & 0.01 & 0.01 & 5.53 & 0.02 & 2.83 & $\begin{array}{l}\mathrm{HCu}- \\
\mathrm{LC}\end{array}$ & $\begin{array}{l}\mathrm{HCu}- \\
\mathrm{HOH}\end{array}$ & $\mathrm{HCu}-\mathrm{HH}$ \\
\hline 2 & 0.32 & 0.65 & 0.00 & 1.53 & 0.02 & 3.95 & 0.01 & 0.00 & 0.00 & 0.00 & 0.03 & 6.48 & 0.03 & 6.46 & $\begin{array}{l}\mathrm{HCu}- \\
\mathrm{LC}\end{array}$ & $\begin{array}{l}\mathrm{HCu}- \\
\mathrm{HOH}\end{array}$ & $\mathrm{HCu}-\mathrm{HH}$ \\
\hline 3 & 0.25 & 0.35 & 0.01 & 0.70 & 0.00 & 4.37 & 0.00 & 0.05 & 0.08 & 0.00 & 0.00 & 5.73 & 0.08 & 7.33 & $\begin{array}{l}\mathrm{HCu}- \\
\mathrm{LC}\end{array}$ & $\begin{array}{l}\mathrm{HCu}- \\
\mathrm{HOH}\end{array}$ & $\mathrm{HCu}-\mathrm{HH}$ \\
\hline 4 & 0.28 & 0.57 & 0.18 & 1.17 & 0.05 & 1.06 & 0.00 & 0.00 & 0.00 & 0.00 & 0.00 & 3.31 & 0.00 & 1.75 & $\begin{array}{l}\mathrm{HCu}- \\
\mathrm{LC}\end{array}$ & $\begin{array}{l}\mathrm{HCu}- \\
\mathrm{LOH}\end{array}$ & $\mathrm{HCu}-\mathrm{HH}$ \\
\hline 5 & 0.46 & 0.66 & 0.00 & 5.68 & 0.04 & 0.68 & 0.02 & 0.15 & 0.27 & 0.01 & 0.03 & 7.69 & 0.31 & 1.70 & $\begin{array}{l}\mathrm{HCu}- \\
\mathrm{LC}\end{array}$ & $\begin{array}{l}\mathrm{HCu}- \\
\mathrm{HOH}\end{array}$ & HCu-LH \\
\hline 6 & 0.09 & 1.20 & 0.00 & 1.52 & 0.01 & 0.23 & 0.00 & 0.00 & 0.00 & 10.29 & 1.67 & 3.05 & 11.96 & 0.41 & $\begin{array}{l}\text { LCu- } \\
\text { HC }\end{array}$ & $\begin{array}{l}\mathrm{LCu}- \\
\mathrm{LOH}\end{array}$ & LCu-HH \\
\hline 7 & 0.13 & 1.02 & 0.00 & 2.00 & 0.03 & 0.03 & 0.01 & 0.00 & 0.00 & 0.01 & 0.03 & 3.22 & 0.04 & 0.05 & $\begin{array}{l}\text { LCu- } \\
\text { LC }\end{array}$ & $\begin{array}{l}\mathrm{LCu}- \\
\mathrm{LOH}\end{array}$ & LCu-HH \\
\hline 8 & 0.02 & 0.47 & 0.00 & 3.6 & 0.01 & 1.16 & 0.01 & 0.00 & 0.00 & 0.00 & 0.01 & 5.27 & 0.01 & 1.90 & $\begin{array}{l}\mathrm{HCu}- \\
\mathrm{LC}\end{array}$ & $\begin{array}{l}\mathrm{HCu}- \\
\mathrm{HOH}\end{array}$ & $\mathrm{HCu}-\mathrm{HH}$ \\
\hline 9 & 0.02 & 0.53 & 0.00 & 3.23 & 0.10 & 0.82 & 0.01 & 0.21 & 0.37 & 18.95 & 2.36 & 4.92 & 21.68 & 2.28 & $\begin{array}{l}\mathrm{HCu}- \\
\mathrm{HC}\end{array}$ & $\begin{array}{l}\mathrm{HCu}- \\
\mathrm{LOH}\end{array}$ & $\mathrm{HCu}-\mathrm{HH}$ \\
\hline 10 & 0.14 & 0.46 & 0.00 & 1.02 & 0.01 & 1.61 & 0.00 & 0.24 & 0.42 & 0.00 & 0.01 & 3.48 & 0.43 & 3.55 & $\begin{array}{l}\mathrm{HCu}- \\
\mathrm{LC}\end{array}$ & $\begin{array}{l}\mathrm{HCu}- \\
\mathrm{LOH}\end{array}$ & $\mathrm{HCu}-\mathrm{HH}$ \\
\hline 11 & 0.23 & 0.43 & 0.09 & 1.06 & 0.01 & 1.93 & 0.00 & 0.00 & 0.00 & 0.00 & 0.00 & 3.75 & 0.00 & 3.15 & $\begin{array}{l}\mathrm{HCu}- \\
\mathrm{LC}\end{array}$ & $\begin{array}{l}\mathrm{HCu}- \\
\mathrm{LOH}\end{array}$ & $\mathrm{HCu}-\mathrm{HH}$ \\
\hline 12 & 0.30 & 0.38 & 0.11 & 0.79 & 0.02 & 1.43 & 0.00 & 0.00 & 0.00 & 0.00 & 0.00 & 3.03 & 0.00 & 2.34 & $\begin{array}{l}\mathrm{HCu}- \\
\mathrm{LC}\end{array}$ & $\begin{array}{l}\mathrm{HCu}- \\
\mathrm{LOH}\end{array}$ & $\mathrm{HCu}-\mathrm{HH}$ \\
\hline 13 & 0.20 & 0.26 & 0.01 & 0.68 & 0.00 & 0.00 & 0.00 & 0.00 & 0.00 & 3.04 & 1.18 & 1.15 & 4.22 & 0.00 & $\begin{array}{l}\text { LCu- } \\
\text { HC }\end{array}$ & $\begin{array}{l}\mathrm{LCu}- \\
\mathrm{LOH}\end{array}$ & LCu-HH \\
\hline 14 & 0.32 & 0.53 & 0.31 & 3.07 & 0.16 & 0.25 & 0.00 & 0.00 & 0.00 & 0.06 & 0.03 & 4.64 & 0.09 & 0.40 & $\begin{array}{l}\text { LCu- } \\
\text { LC }\end{array}$ & $\begin{array}{l}\mathrm{LCu}- \\
\mathrm{LOH}\end{array}$ & LCu-HH \\
\hline 15 & 0.72 & 1.79 & 0.01 & 2.48 & 0.00 & 0.00 & 0.00 & 0.00 & 0.00 & 2.26 & 1.53 & 5.00 & 3.79 & 0.00 & $\begin{array}{l}\mathrm{LCu}- \\
\mathrm{HC}\end{array}$ & $\begin{array}{l}\mathrm{LCu}- \\
\mathrm{HOH}\end{array}$ & LCu-LH \\
\hline 16 & 0.01 & 0.74 & 0.00 & 3.46 & 0.00 & 0.08 & 0.01 & 0.00 & 0.00 & 0.00 & 0.00 & 4.30 & 0.00 & 0.14 & $\begin{array}{l}\text { LCu- } \\
\text { LC }\end{array}$ & $\begin{array}{l}\mathrm{LCu}- \\
\mathrm{LOH}\end{array}$ & LCu-LH \\
\hline 17 & 0.00 & 0.04 & 0.00 & 5.85 & 0.01 & 0.72 & 0.01 & 0.06 & 0.10 & 0.01 & 0.00 & 6.69 & 0.11 & 1.41 & $\begin{array}{l}\mathrm{HCu}- \\
\mathrm{LC}\end{array}$ & $\begin{array}{l}\mathrm{HCu}- \\
\mathrm{HOH}\end{array}$ & HCu-LH \\
\hline
\end{tabular}




\section{ELSEVIER_MINE_4780}

\begin{tabular}{|c|c|c|c|c|c|c|c|c|c|c|c|c|c|c|c|c|c|}
\hline 18 & 1.51 & 0.36 & 0.00 & 0.56 & 0.00 & 0.06 & 0.00 & 0.00 & 0.00 & 0.65 & 0.37 & 2.49 & 1.02 & 0.09 & $\begin{array}{l}\text { LCU- } \\
\text { LC }\end{array}$ & $\begin{array}{l}\text { LCu- } \\
\text { LOH }\end{array}$ & LCu-LH \\
\hline 19 & 0.84 & 1.39 & 0.00 & 1.23 & 0.02 & 0.09 & 0.01 & 0.00 & 0.00 & 9.76 & 1.42 & 3.58 & 11.18 & 0.15 & $\begin{array}{l}\text { LCu- } \\
\mathrm{HC}\end{array}$ & $\begin{array}{l}\text { LCu- } \\
\text { LOH }\end{array}$ & LCu-LH \\
\hline 20 & 0.14 & 0.79 & 0.00 & 8.33 & 0.02 & 0.17 & 0.01 & 0.02 & 0.04 & 0.02 & 0.00 & 9.48 & 0.06 & 0.36 & $\begin{array}{l}\text { LCu- } \\
\text { LC }\end{array}$ & $\begin{array}{l}\mathrm{LCu}- \\
\mathrm{HOH}\end{array}$ & LCu-LH \\
\hline 21 & 1.61 & 0.37 & 0.82 & 0.96 & 0.02 & 0.01 & 0.00 & 0.00 & 0.00 & 0.00 & 0.00 & 3.79 & 0.00 & 0.02 & $\begin{array}{l}\text { LCu- } \\
\text { LC }\end{array}$ & $\begin{array}{l}\text { LCu- } \\
\text { LOH }\end{array}$ & LCu-H \\
\hline 22 & 0.02 & 0.55 & 0.00 & 2.57 & 0.01 & 0.02 & 0.02 & 0.00 & 0.00 & 0.01 & 0.01 & 3.19 & 0.02 & 0.03 & $\begin{array}{l}\text { LCu- } \\
\text { LC }\end{array}$ & $\begin{array}{l}\text { LCu- } \\
\text { LOH }\end{array}$ & LCu-LH \\
\hline 23 & 0.00 & 0.14 & 0.00 & 7.72 & 0.07 & 0.01 & 0.01 & 0.00 & 0.00 & 0.02 & 0.01 & 7.95 & 0.03 & 0.02 & $\begin{array}{l}\text { LCu- } \\
\text { LC }\end{array}$ & $\begin{array}{l}\mathrm{LCu}- \\
\mathrm{HOH}\end{array}$ & LCu-LH \\
\hline 24 & 1.84 & 0.27 & 0.00 & 0.30 & 0.00 & 0.25 & 0.00 & 0.10 & 0.18 & 0.00 & 0.01 & 2.76 & 0.19 & 0.80 & $\begin{array}{l}\text { HCu- } \\
\text { LC }\end{array}$ & $\begin{array}{l}\mathrm{HCu}- \\
\mathrm{LOH}\end{array}$ & HCu-Lr \\
\hline 25 & 0.01 & 0.26 & 0.00 & 4.04 & 0.03 & 0.07 & 0.01 & 0.00 & 0.00 & 11.28 & 0.26 & 4.42 & 11.54 & 0.12 & $\begin{array}{l}\text { LCu- } \\
\text { HC }\end{array}$ & $\begin{array}{l}\text { LCu- } \\
\text { LOH }\end{array}$ & LCu-LH \\
\hline 26 & 0.35 & 0.66 & 0.00 & 6.02 & 0.03 & 0.68 & 0.02 & 0.19 & 0.33 & 0.01 & 0.02 & 7.95 & 0.36 & 1.81 & $\begin{array}{l}\text { HCu- } \\
\text { LC }\end{array}$ & $\begin{array}{l}\mathrm{HCu}- \\
\mathrm{HOH}\end{array}$ & HCu-Lr \\
\hline 27 & 0.90 & 0.40 & 0.01 & 0.51 & 0.03 & 0.00 & 0.01 & 0.00 & 0.00 & 17.32 & 0.16 & 1.86 & 17.48 & 0.00 & $\begin{array}{l}\text { LCu- } \\
\text { HC }\end{array}$ & $\begin{array}{l}\text { LCu- } \\
\text { LOH }\end{array}$ & LCu-LH \\
\hline 28 & 0.00 & 0.07 & 0.00 & 8.02 & 0.05 & 0.34 & 0.03 & 0.10 & 0.17 & 0.03 & 0.01 & 8.61 & 0.21 & 0.92 & $\begin{array}{l}\mathrm{HCu}- \\
\mathrm{LC}\end{array}$ & $\begin{array}{l}\mathrm{HCu}- \\
\mathrm{HOH}\end{array}$ & HCu-Lr \\
\hline 29 & 1.27 & 0.24 & 0.01 & 0.29 & 0.03 & 0.04 & 0.01 & 0.00 & 0.00 & 23.27 & 0.47 & 1.89 & 23.74 & 0.07 & $\begin{array}{l}\text { LCu- } \\
\text { HC }\end{array}$ & $\begin{array}{l}\text { LCu- } \\
\text { LOH }\end{array}$ & LCu-LH \\
\hline 30 & 0.01 & 0.28 & 0.00 & 8.06 & 0.16 & 0.02 & 0.01 & 0.00 & 0.00 & 0.01 & 0.01 & 8.54 & 0.02 & 0.03 & $\begin{array}{l}\text { LCu- } \\
\text { LC }\end{array}$ & $\begin{array}{l}\mathrm{LCu}- \\
\mathrm{HOH}\end{array}$ & LCu-LH \\
\hline 31 & 0.14 & 0.46 & 0.00 & 10.29 & 0.02 & 0.06 & 0.03 & 0.00 & 0.00 & 0.03 & 0.02 & 11.00 & 0.05 & 0.09 & $\begin{array}{l}\text { LCu- } \\
\text { LC }\end{array}$ & $\begin{array}{l}\mathrm{LCu}- \\
\mathrm{HOH}\end{array}$ & LCu-LH \\
\hline 32 & 0.02 & 0.09 & 0.00 & 7.94 & 0.01 & 0.42 & 0.17 & 0.14 & 0.25 & 0.21 & 0.07 & 8.79 & 0.53 & 1.23 & $\begin{array}{l}\mathrm{HCu}- \\
\mathrm{LC}\end{array}$ & $\begin{array}{l}\mathrm{HCu}- \\
\mathrm{HOH}\end{array}$ & HCu-Lr \\
\hline 33 & 0.96 & 1.28 & 0.04 & 1.20 & 0.01 & 0.00 & 0.00 & 0.00 & 0.00 & 4.80 & 1.24 & 3.49 & 6.04 & 0.00 & $\begin{array}{l}\text { LCu- } \\
\text { HC }\end{array}$ & $\begin{array}{l}\text { LCu- } \\
\text { LOH }\end{array}$ & LCu-LH \\
\hline 34 & 0.00 & 0.13 & 0.00 & 8.35 & 0.02 & 0.05 & 0.01 & 0.00 & 0.00 & 0.03 & 0.01 & 8.56 & 0.04 & 0.07 & $\begin{array}{l}\text { LCu- } \\
\text { LC }\end{array}$ & $\begin{array}{l}\mathrm{LCu}- \\
\mathrm{HOH}\end{array}$ & LCu-LH \\
\hline 35 & 0.14 & 0.57 & 0.00 & 4.36 & 0.00 & 0.62 & 0.02 & 0.13 & 0.23 & 16.2 & 2.38 & 5.84 & 18.81 & 1.71 & $\begin{array}{l}\mathrm{HCu}- \\
\mathrm{HC}\end{array}$ & $\begin{array}{l}\mathrm{HCu}- \\
\mathrm{HOH}\end{array}$ & HCu-Lr \\
\hline 36 & 0.05 & 0.95 & 0.00 & 3.73 & 0.00 & 0.00 & 0.01 & 0.00 & 0.00 & 2.26 & 0.07 & 4.74 & 2.33 & 0.01 & $\begin{array}{l}\mathrm{LCu}- \\
\mathrm{HC}\end{array}$ & $\begin{array}{l}\text { LCu- } \\
\text { LOH }\end{array}$ & LCu-LH \\
\hline 37 & 0.01 & 1.23 & 0.00 & 10.02 & 0.00 & 0.17 & 0.01 & 0.00 & 0.00 & 0.00 & 0.00 & 11.44 & 0.00 & 0.28 & $\begin{array}{l}\text { LCu- } \\
\text { LC }\end{array}$ & $\begin{array}{l}\mathrm{LCu}- \\
\mathrm{HOH}\end{array}$ & LCu-LH \\
\hline 38 & 0.12 & 3.20 & 0.00 & 1.80 & 0.01 & 0.04 & 0.01 & 0.00 & 0.00 & 0.02 & 0.01 & 5.18 & 0.03 & 0.06 & $\begin{array}{l}\text { LCu- } \\
\text { LC }\end{array}$ & $\begin{array}{l}\mathrm{LCu}- \\
\mathrm{HOH}\end{array}$ & LCu-LH \\
\hline
\end{tabular}




\section{ELSEVIER_MINE_4780}

\begin{tabular}{|c|c|c|c|c|c|c|c|c|c|c|c|c|c|c|c|c|c|}
\hline 39 & 0.01 & 0.80 & 0.00 & 10.47 & 0.00 & 0.20 & 0.02 & 0.00 & 0.00 & 0.01 & 0.00 & 11.5 & 0.01 & 0.33 & $\begin{array}{l}\text { LCu- } \\
\text { LC }\end{array}$ & $\begin{array}{l}\mathrm{LCu}- \\
\mathrm{HOH}\end{array}$ & LCu-LH \\
\hline 40 & 0.01 & 0.12 & 0.00 & 4.48 & 0.06 & 0.18 & 0.00 & 0.00 & 0.00 & 0.01 & 0.00 & 4.85 & 0.01 & 0.3 & $\begin{array}{l}\text { LCu- } \\
\text { LC }\end{array}$ & $\begin{array}{l}\text { LCu- } \\
\text { LOH }\end{array}$ & LCu-LH \\
\hline 41 & 0.00 & 0.23 & 0.00 & 5.30 & 0.62 & 0.00 & 0.01 & 0.00 & 0.00 & 7.85 & 3.51 & 6.16 & 11.36 & 0.00 & $\begin{array}{l}\text { LCu- } \\
\text { HC }\end{array}$ & $\begin{array}{l}\mathrm{LCu}- \\
\mathrm{HOH}\end{array}$ & LCu-LH \\
\hline 42 & 0.52 & 0.93 & 0.00 & 1.04 & 0.02 & 0.06 & 0.01 & 0.00 & 0.00 & 24.29 & 0.33 & 2.58 & 24.62 & 0.09 & $\begin{array}{l}\text { LCu- } \\
\text { HC }\end{array}$ & $\begin{array}{l}\text { LCu- } \\
\text { LOH }\end{array}$ & LCu-LH \\
\hline 43 & 0.42 & 0.66 & 0.03 & 1.43 & 0.10 & 0.01 & 0.01 & 0.00 & 0.00 & 11.06 & 0.24 & 2.66 & 11.30 & 0.01 & $\begin{array}{l}\text { LCu- } \\
\text { HC }\end{array}$ & $\begin{array}{l}\text { LCu- } \\
\text { LOH }\end{array}$ & LCu-LH \\
\hline 44 & 0.02 & 0.25 & 0.00 & 9.30 & 0.00 & 0.03 & 0.02 & 0.00 & 0.00 & 6.06 & 0.15 & 9.62 & 6.21 & 0.05 & $\begin{array}{l}\text { LCu- } \\
\text { HC }\end{array}$ & $\begin{array}{l}\mathrm{LCu}- \\
\mathrm{HOH}\end{array}$ & LCu-LH \\
\hline 45 & 0.01 & 0.55 & 0.00 & 3.56 & 0.00 & 0.11 & 0.01 & 0.00 & 0.00 & 5.13 & 0.22 & 4.24 & 5.35 & 0.18 & $\begin{array}{l}\text { LCu- } \\
\text { HC }\end{array}$ & $\begin{array}{l}\text { LCu- } \\
\text { LOH }\end{array}$ & LCu-LH \\
\hline 46 & 0.71 & 0.31 & 0.02 & 1.11 & 0.12 & 0.03 & 0.01 & 0.00 & 0.00 & 15.47 & 0.30 & 2.31 & 15.77 & 0.04 & $\begin{array}{l}\text { LCu- } \\
\text { HC }\end{array}$ & $\begin{array}{l}\text { LCu- } \\
\text { LOH }\end{array}$ & LCu-LH \\
\hline 47 & 0.01 & 0.21 & 0.00 & 7.11 & 0.01 & 0.28 & 0.01 & 0.02 & 0.03 & 13.57 & 1.41 & 7.65 & 15.01 & 0.54 & $\begin{array}{l}\mathrm{HCu}- \\
\mathrm{HC}\end{array}$ & $\begin{array}{l}\mathrm{HCu}- \\
\mathrm{HOH}\end{array}$ & HCu-LH \\
\hline 48 & 0.14 & 0.69 & 0.00 & 1.52 & 0.00 & 0.85 & 0.00 & 0.00 & 0.00 & 1.16 & 0.02 & 3.20 & 1.18 & 1.40 & $\begin{array}{l}\mathrm{HCu}- \\
\mathrm{LC}\end{array}$ & $\begin{array}{l}\mathrm{HCu}- \\
\mathrm{LOH}\end{array}$ & $\mathrm{HCu}-\mathrm{HH}$ \\
\hline 49 & 1.10 & 0.26 & 0.01 & 0.37 & 0.06 & 0.08 & 0.01 & 0.00 & 0.00 & 25.25 & 0.75 & 1.89 & 26.00 & 0.14 & $\begin{array}{l}\text { LCu- } \\
\text { HC }\end{array}$ & $\begin{array}{l}\text { LCu- } \\
\text { LOH }\end{array}$ & LCu-LH \\
\hline 50 & 0.02 & 0.19 & 0.00 & 3.85 & 0.00 & 7.05 & 0.01 & 0.00 & 0.00 & 0.00 & 0.14 & 11.12 & 0.14 & 11.54 & $\begin{array}{l}\mathrm{HCu}- \\
\mathrm{LC}\end{array}$ & $\begin{array}{l}\mathrm{HCu}- \\
\mathrm{HOH}\end{array}$ & HCu-LH \\
\hline 51 & 0.18 & 1.12 & 0.00 & 1.62 & 0.02 & 0.61 & 0.00 & 0.00 & 0.00 & 17.41 & 0.08 & 3.55 & 17.49 & 1.00 & $\begin{array}{l}\mathrm{HCu}- \\
\mathrm{HC}\end{array}$ & $\begin{array}{l}\mathrm{HCu}- \\
\mathrm{LOH}\end{array}$ & HCu-LH \\
\hline 52 & 0.85 & 0.75 & 0.00 & 1.10 & 0.01 & 0.01 & 0.00 & 0.00 & 0.00 & 15.00 & 1.26 & 2.72 & 16.26 & 0.01 & $\begin{array}{l}\text { LCu- } \\
\text { HC }\end{array}$ & $\begin{array}{l}\text { LCu- } \\
\text { LOH }\end{array}$ & LCu-HH \\
\hline 53 & 1.89 & 0.18 & 0.01 & 0.24 & 0.03 & 0.01 & 0.01 & 0.00 & 0.00 & 17.79 & 0.10 & 2.37 & 17.89 & 0.01 & $\begin{array}{l}\text { LCu- } \\
\text { HC }\end{array}$ & $\begin{array}{l}\text { LCu- } \\
\text { LOH }\end{array}$ & LCu-LH \\
\hline 54 & 0.18 & 0.66 & 0.01 & 6.17 & 0.07 & 0.04 & 0.00 & 0.00 & 0.00 & 9.93 & 1.03 & 7.13 & 10.96 & 0.06 & $\begin{array}{l}\text { LCu- } \\
\text { HC }\end{array}$ & $\begin{array}{l}\mathrm{LCu}- \\
\mathrm{HOH}\end{array}$ & LCu-LH \\
\hline 55 & 1.61 & 0.99 & 0.01 & 1.24 & 0.04 & 1.08 & 0.02 & 0.34 & 0.60 & 7.78 & 3.08 & 5.33 & 11.46 & 3.28 & $\begin{array}{l}\mathrm{HCu}- \\
\mathrm{HC}\end{array}$ & $\begin{array}{l}\mathrm{HCu}- \\
\mathrm{HOH}\end{array}$ & HCu-LH \\
\hline 56 & 1.03 & 1.07 & 0.00 & 2.77 & 0.04 & 0.03 & 0.00 & 0.00 & 0.00 & 17.1 & 0.46 & 4.94 & 17.56 & 0.05 & $\begin{array}{l}\text { LCu- } \\
\text { HC }\end{array}$ & $\begin{array}{l}\text { LCu- } \\
\text { LOH }\end{array}$ & LCu-LH \\
\hline 57 & 0.84 & 0.78 & 0.02 & 1.92 & 0.1 & 1.31 & 0.01 & 0.11 & 0.19 & 10.24 & 3.75 & 5.09 & 14.18 & 2.77 & $\begin{array}{l}\mathrm{HCu}- \\
\mathrm{HC}\end{array}$ & $\begin{array}{l}\mathrm{HCu}- \\
\mathrm{HOH}\end{array}$ & HCu-LH \\
\hline 58 & 0.07 & 0.50 & 0.03 & 1.07 & 0.04 & 0.00 & 0.00 & 0.00 & 0.00 & 0.00 & 0.01 & 1.71 & 0.01 & 0.00 & $\begin{array}{l}\text { LCu- } \\
\text { LC }\end{array}$ & $\begin{array}{l}\text { LCu- } \\
\text { LOH }\end{array}$ & LCu-HH \\
\hline 59 & 0.39 & 1.43 & 0.00 & 2.27 & 0.03 & 0.02 & 0.01 & 0.00 & 0.00 & 0.01 & 0.03 & 4.15 & 0.04 & 0.03 & $\begin{array}{l}\text { LCu- } \\
\text { LC }\end{array}$ & $\begin{array}{l}\text { LCu- } \\
\text { LOH }\end{array}$ & LCu-HH \\
\hline
\end{tabular}




\section{ELSEVIER_MINE_4780}

\begin{tabular}{l|l|l|l|l}
60 & 0.11 & 1.01 & 0.01
\end{tabular}

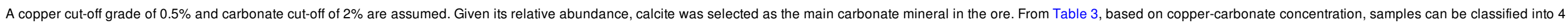

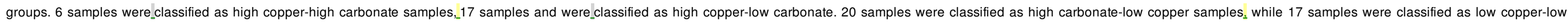
carbonate samples.

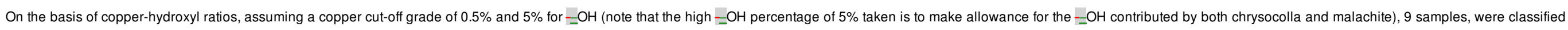
as high copper-low -OH. 14 samples as high copper-high _-OH, while 12 samples were_classified as low copper-high - $-\mathrm{OH} .25$ samples were classified as low copper-low-OH samples (Table 3).

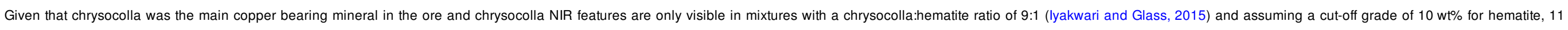
samples were_classified as high copper-high hematite, 12 samples as high copper-low hematite, 8 samples as high hematite-low copper and 29 samples as low copper-low hematite samples (Table 3).

Comparing the various classifications above, the following groups and samples could be considered for economic preconcentration:

i. High copper-low carbonate (sample no. 1, 2, 3, 4, 5, 8, 10,11, 12, 17, 24, 26, 28 32, 48, 50 and 60).

ii. High copper-low - $\mathrm{OH}$ (sample no. 4, 9, 10,11, 12, 24, 48, 51 and 60).

iii. High copper-high hematite (sample no. 1, 2, 3, 4, 8, 9, 10,11, 12, 48 and 60) and

iv. High copper-low hematite (sample no. 5, 17, 24, 26, 28, 32, 35, 47, 50, 51, 55 and 57).

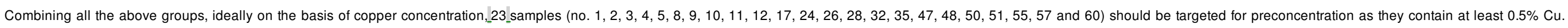
However, some of these samples contain either high $\mathrm{CO}_{3}{ }^{2-}$ and/or high $-\mathrm{OH}$, which is undesirable in downstream leaching.

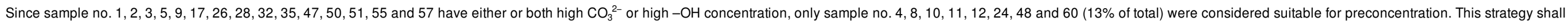
be referred to as Func. (1).

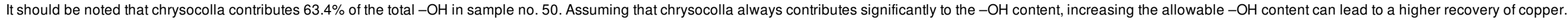

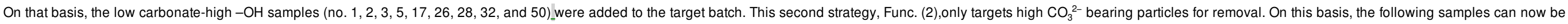
targeted: no. $1,2,3,4,5,8,10,11,12,17,24,26,28,32,48,50$ and 60 . This increases the yield to $28 \%$ of the particles at the cost of increasing dilution.

\subsection{Samples NIR spectral correlation and interpretation}

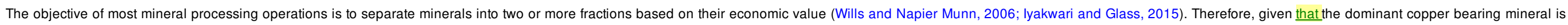
chrysocolla with minor malachite, samples are classified based on their NIR spectra signature with reference to strategies by lyakwari and Glass (2015). Two strategies were developed.

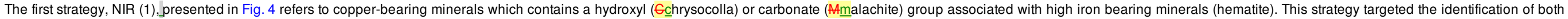
calcite and clay (muscovite/kaolinite) particles for later removal by assuming that hematite completely masks chrysocolla features. On this basis samples are classified into three groups as:

i. Products - the samples with all NIR spectra showing chrysocolla and _or hematite, chlorite, biotite pattern. This group includes featureless NIR spectra.

ii. Waste - the samples with all NIR spectra showing calcite and or muscovite characteristic spectra.

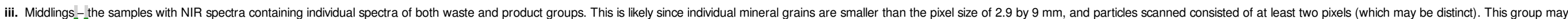
require further liberation to a size not less than the pixel size and rescanned. 


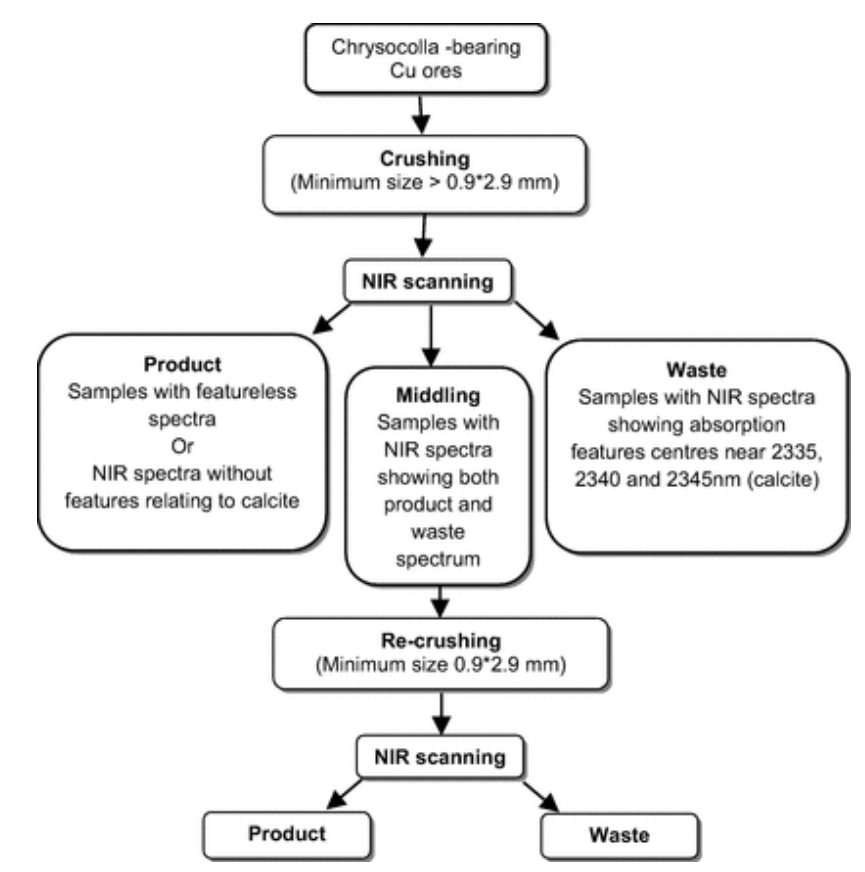

Fig. 5 Copper samples preconcentration strategy NIR (2) targeted at eliminating only calcite-bearing samples as waste (modified from lyakwari and Glass, 2015).

\subsubsection{Individual sample description}

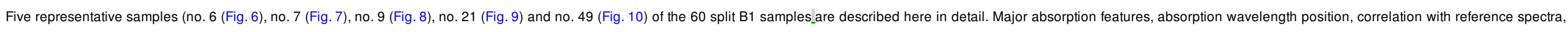
and the possible mineral(s) responsible for the absorption features are correlated with mineral data and field scan images. Comparison of classification with PXRF data is presented in Table 4. 


\section{ELSEVIER_MINE_4780}
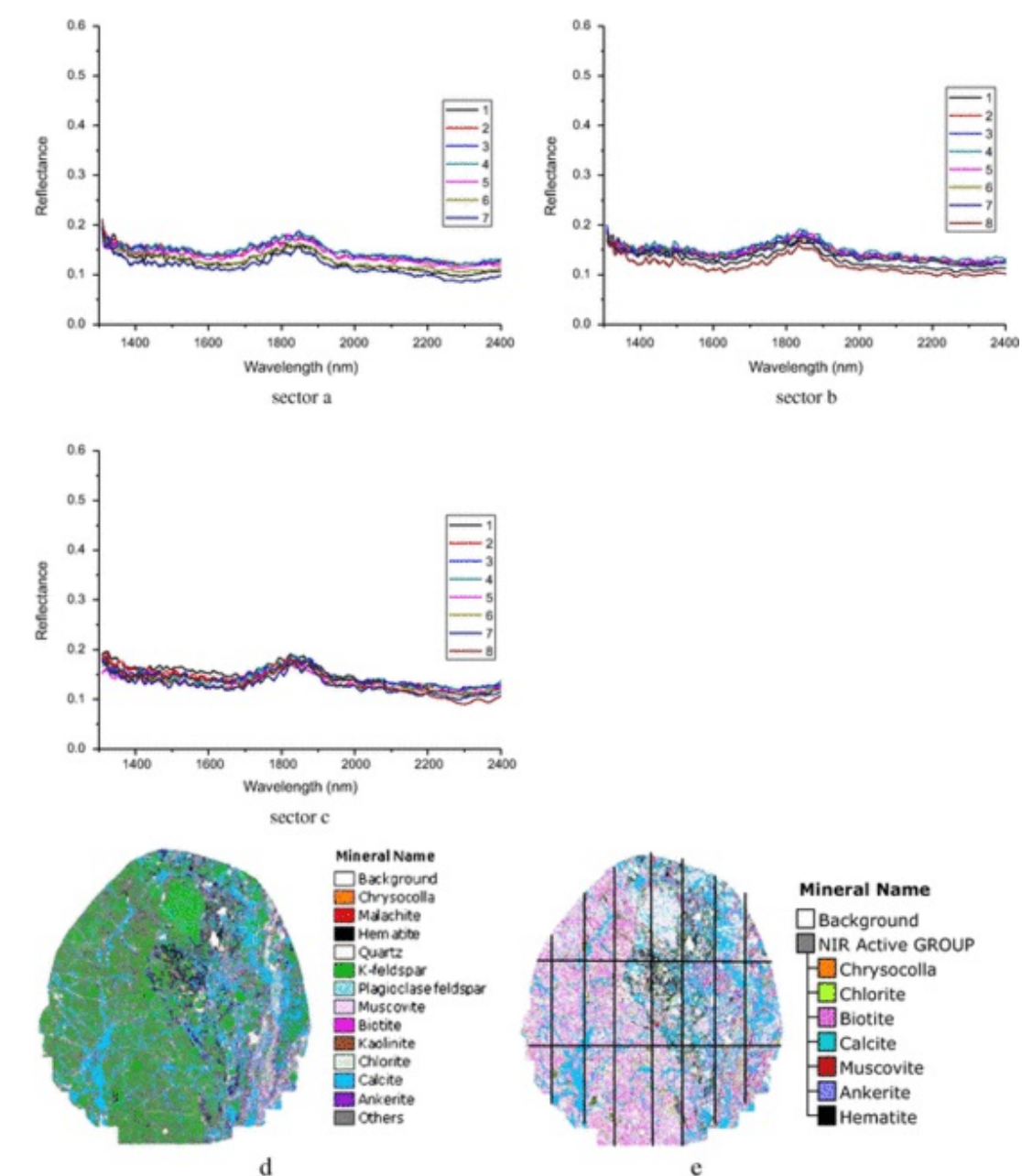

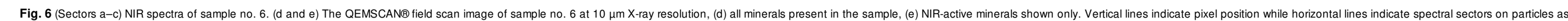
scanned. 



\section{ELSEVIER_MINE_4780}
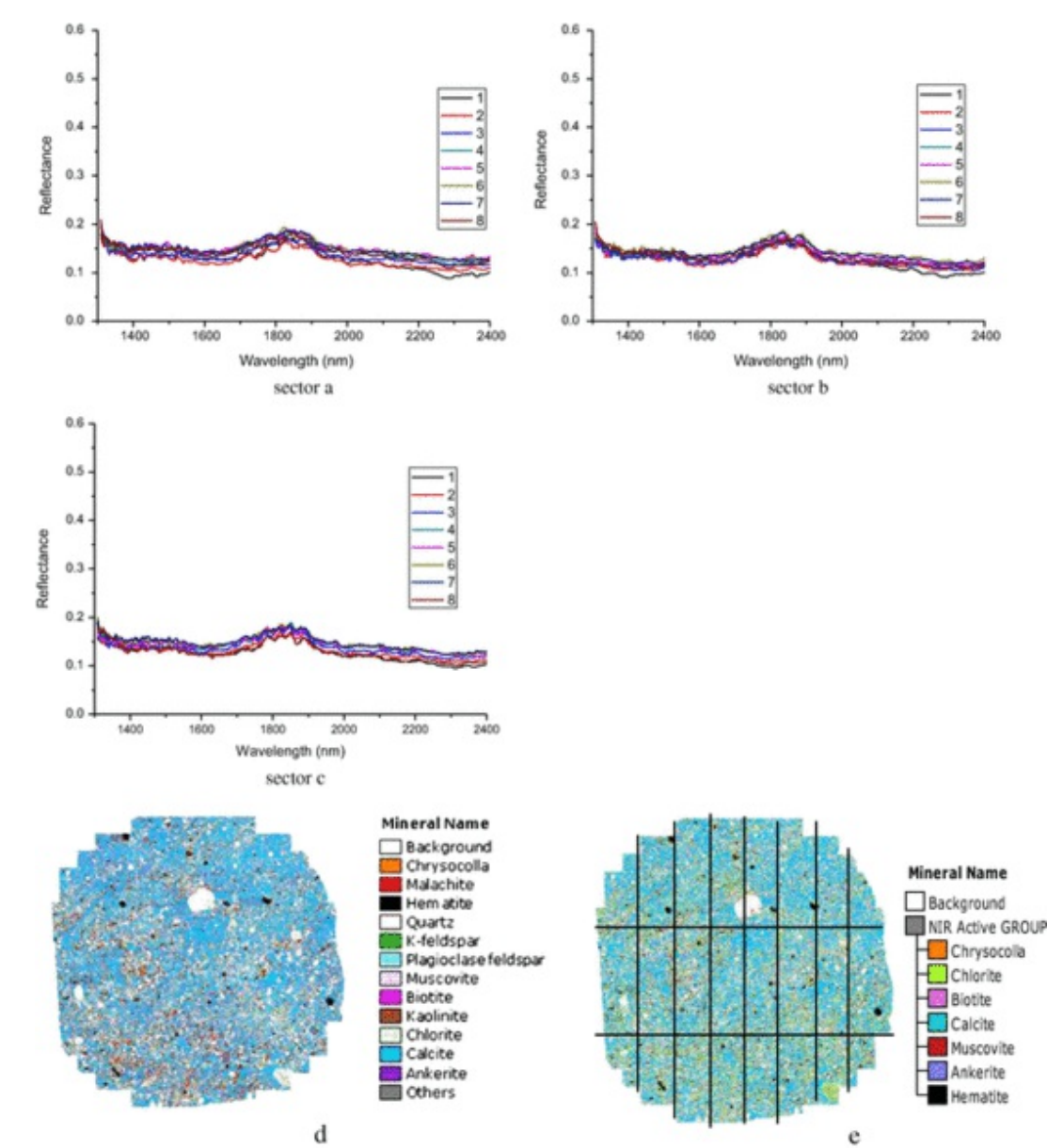

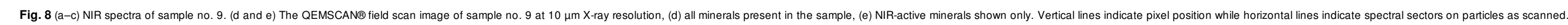





\section{ELSEVIER_MINE_4780}
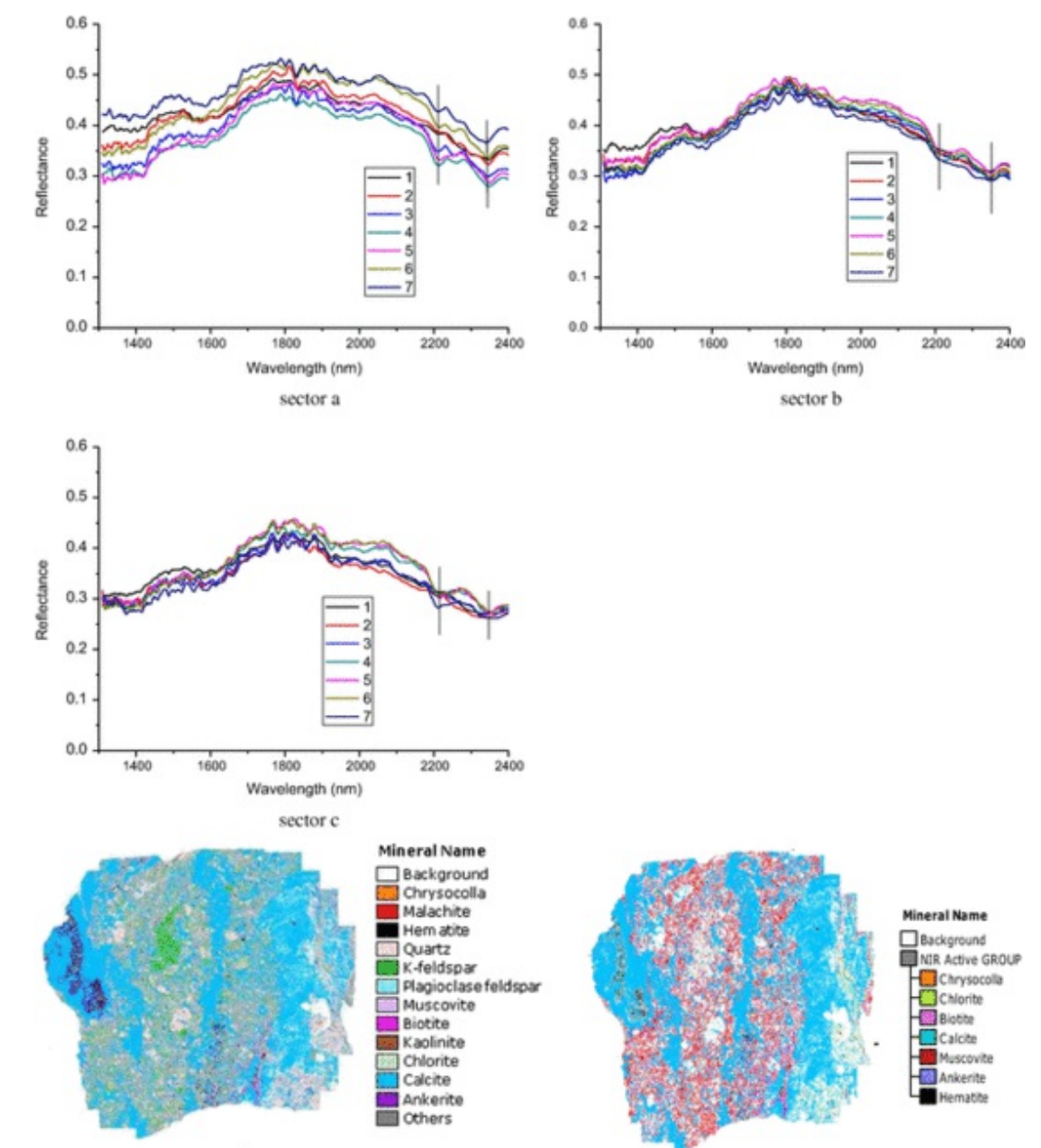

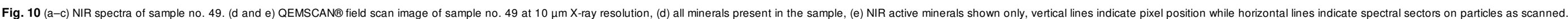

Table 4 Classifications of split B1 samples, where $\mathrm{P}, \mathrm{M}$ and $\mathrm{W}$ indicate product, middling and waste, respectively, $\mathrm{M}^{*}$ and $\mathrm{M}$-indicates middling with more product or waste pixels respectively.

\begin{tabular}{|c|c|c|c|c|c|c|c|c|}
\hline \multirow[t]{2}{*}{ Sample ID } & \multirow[t]{2}{*}{ Cu Content (Qem)\% } & \multirow[t]{2}{*}{ Cu Content (PXRF)\% } & \multicolumn{6}{|c|}{ Classification } \\
\hline & & & $\mathrm{NIR}(1)$ & $\operatorname{NIR}(2)$ & QEMSCAN & PXRF & Func(1) & Func(2) \\
\hline & & & $P$ & $P$ & $\mathrm{P}$ & $P$ & w & $\mathrm{P}$ \\
\hline & & & $P$ & $P$ & $P$ & $P$ & W & $P$ \\
\hline 2 & 6.46 & 2.59 & $P$ & $\mathrm{P}$ & $\mathrm{P}$ & $P$ & W & $P$ \\
\hline 3 & 7.33 & 7.74 & $P$ & $P$ & $P$ & $P$ & W & $P$ \\
\hline 4 & 1.75 & 1.37 & $P$ & $P$ & $\mathrm{P}$ & $P$ & $\mathrm{P}$ & $\mathrm{P}$ \\
\hline 5 & 1.70 & 1.77 & $\mathrm{P}$ & $P$ & $\mathrm{P}$ & $P$ & w & $P$ \\
\hline
\end{tabular}




\section{ELSEVIER_MINE_4780}

\begin{tabular}{|c|c|c|c|c|c|c|c|c|}
\hline 6 & 0.41 & 0.58 & $P$ & $P$ & $w$ & $P$ & w & w \\
\hline 7 & 0.05 & 0.12 & $P$ & $P$ & $w$ & w & w & $P$ \\
\hline 8 & 1.90 & 0.61 & $P$ & $P$ & P & $P$ & $P$ & $P$ \\
\hline 9 & 2.28 & 1.89 & $P$ & $P$ & $P$ & $P$ & w & w \\
\hline 10 & 3.55 & 1.05 & $M^{*}(P)$ & $P$ & $P$ & $P$ & $\mathrm{P}$ & $P$ \\
\hline 11 & 3.15 & 3.93 & $M^{*}(\mathrm{P})$ & $P$ & $P$ & $P$ & $P$ & $P$ \\
\hline 12 & 2.34 & 1.98 & $P$ & $P$ & $P$ & $P$ & $P$ & $P$ \\
\hline 13 & 0.00 & 0.03 & $M^{*}(\mathrm{P})$ & $P$ & w & W & W & w \\
\hline 14 & 0.40 & 0.97 & $M-(W)$ & $P$ & w & $P$ & w & $P$ \\
\hline 15 & 0.00 & 0.03 & $M-(W)$ & $P$ & w & w & w & w \\
\hline 16 & 0.14 & 0.12 & $M-(W)$ & $M-(W)$ & w & w & w & $P$ \\
\hline 17 & 1.41 & 0.72 & $P$ & $P$ & $P$ & $P$ & w & $P$ \\
\hline 18 & 0.09 & 0.04 & w & $P$ & w & w & w & $P$ \\
\hline 19 & 0.15 & 0.32 & $P$ & $P$ & $w$ & w & w & w \\
\hline 20 & 0.36 & 0.12 & $P$ & $P$ & w & w & w & $P$ \\
\hline 21 & 0.02 & 0.10 & $M-(W)$ & $P$ & $w$ & W & w & $P$ \\
\hline 22 & 0.03 & 0.03 & P & $P$ & w & w & w & $P$ \\
\hline 23 & 0.02 & 0.04 & P & P & w & w & w & P \\
\hline 24 & 0.80 & 0.15 & w & $P$ & $P$ & w & $P$ & $P$ \\
\hline 25 & 0.12 & 0.13 & $P$ & $P$ & w & w & w & w \\
\hline 26 & 1.81 & 2.02 & $P$ & $P$ & $P$ & $P$ & w & $P$ \\
\hline 27 & 0.00 & 0.02 & w & $P$ & w & w & w & w \\
\hline 28 & 0.92 & 0.75 & $P$ & $P$ & $P$ & $P$ & w & $P$ \\
\hline 29 & 0.07 & 0.06 & w & $P$ & w & w & w & w \\
\hline 30 & 0.03 & 0.07 & P & $P$ & w & w & w & P \\
\hline 31 & 0.09 & 0.09 & P & $P$ & w & w & w & $P$ \\
\hline 32 & 1.23 & 0.34 & P & P & P & w & w & P \\
\hline 33 & 0.00 & 0.02 & $M-(W)$ & $P$ & w & W & w & w \\
\hline 34 & 0.07 & 0.05 & $P$ & $P$ & w & w & w & $P$ \\
\hline 35 & 1.71 & 2.16 & M-(W) & $M-(W)$ & $P$ & $P$ & w & w \\
\hline 36 & 0.01 & 0.03 & $M-(W)$ & $P$ & w & w & w & w \\
\hline 37 & 0.28 & 0.17 & $P$ & $P$ & $w$ & w & w & $P$ \\
\hline
\end{tabular}




\section{ELSEVIER_MINE_4780}

\begin{tabular}{|c|c|c|c|c|c|c|c|c|}
\hline 38 & 0.06 & 0.22 & $P$ & $P$ & $w$ & w & $w$ & $P$ \\
\hline 39 & 0.33 & 0.16 & $\mathrm{P}$ & $P$ & $w$ & w & $w$ & P \\
\hline 40 & 0.30 & 0.15 & $\mathrm{P}$ & $P$ & $w$ & w & w & $\mathrm{P}$ \\
\hline 41 & 0.00 & 0.04 & $\mathrm{P}$ & $P$ & $w$ & w & w & w \\
\hline 42 & 0.09 & 0.11 & $M-(W)$ & $P$ & $w$ & w & $w$ & w \\
\hline 43 & 0.01 & 0.08 & w & $M-(W)$ & w & w & w & w \\
\hline 44 & 0.05 & 0.08 & $\mathrm{P}$ & $P$ & w & w & w & w \\
\hline 45 & 0.18 & 0.11 & $\mathrm{P}$ & $P$ & $w$ & w & $w$ & w \\
\hline 46 & 0.04 & 0.08 & $M-(W)$ & $M-(W)$ & w & w & $w$ & w \\
\hline 47 & 0.54 & 0.27 & $M-(W)$ & $M-(W)$ & $P$ & w & w & w \\
\hline 48 & 1.40 & 1.44 & $\mathrm{P}$ & $P$ & $\mathrm{P}$ & $P$ & $P$ & P \\
\hline 49 & 0.14 & 0.09 & w & w & $w$ & w & w & w \\
\hline 50 & 11.54 & 4.08 & $\mathrm{P}$ & $P$ & $P$ & $P$ & w & P \\
\hline 51 & 1.00 & 1.49 & $\mathrm{P}$ & $P$ & $P$ & $P$ & $w$ & w \\
\hline 52 & 0.01 & 0.14 & $\mathrm{M}^{*}(\mathrm{P})$ & $P$ & $w$ & w & $w$ & w \\
\hline 53 & 0.01 & 0.05 & w & $P$ & $w$ & w & $w$ & w \\
\hline 54 & 0.06 & 0.35 & $M-(W)$ & $P$ & $w$ & w & $w$ & w \\
\hline 55 & 3.28 & 7.64 & w & $P$ & $P$ & $P$ & w & w \\
\hline 56 & 0.05 & 0.05 & w & w & $w$ & w & $w$ & w \\
\hline 57 & 2.77 & 4.87 & w & $P$ & $\mathrm{P}$ & $P$ & $w$ & w \\
\hline 58 & 0.00 & 0.05 & $\mathrm{P}$ & $P$ & $w$ & w & w & $\mathrm{P}$ \\
\hline 59 & 0.03 & 0.20 & $\mathrm{P}$ & $P$ & $w$ & w & $w$ & P \\
\hline 60 & 1.98 & 1.96 & $P$ & $P$ & $P$ & $P$ & $P$ & $P$ \\
\hline
\end{tabular}

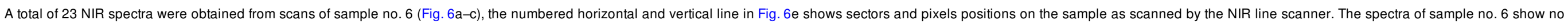

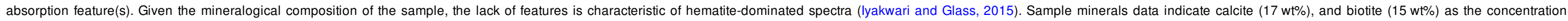

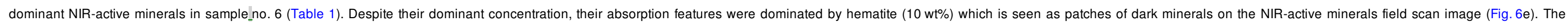

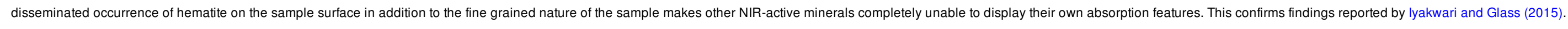

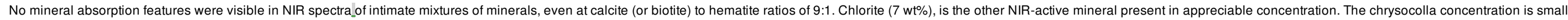
at $1 \mathrm{wt} \%$.

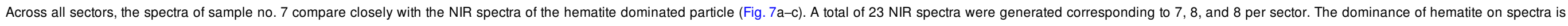

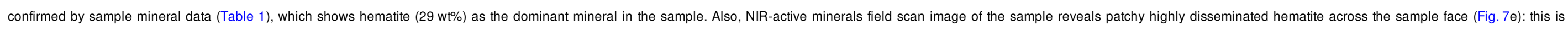

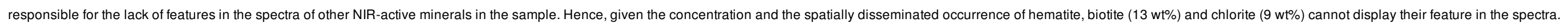

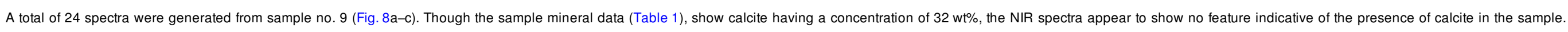




\section{ELSEVIER_MINE_4780}

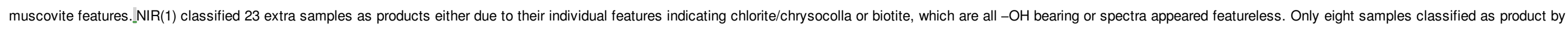
Func. (1), out of which only sample no. 24 was classified waste by NIR (1).

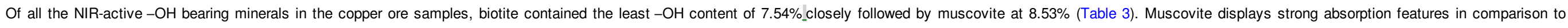

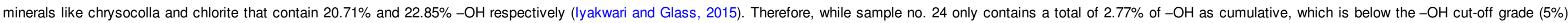

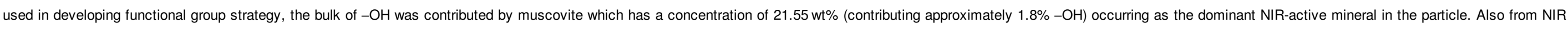

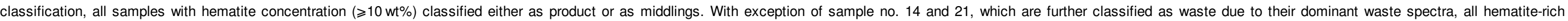
middling were classified as product.

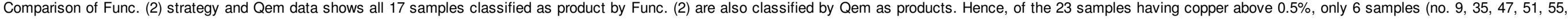

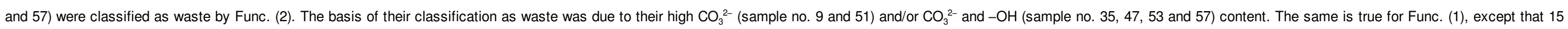
of the 23 Qem products were classified as waste by Func. (1).

\subsection{Validation of NIR strategies}

\subsubsection{Splits $B 2$}

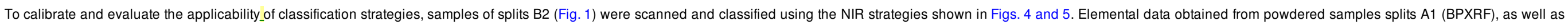

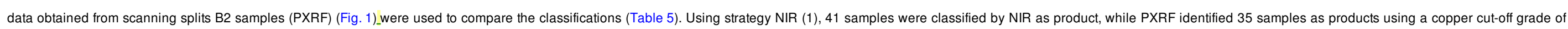

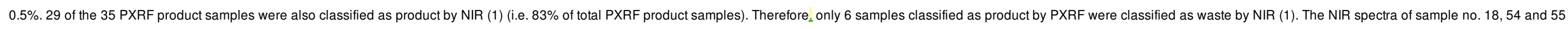
shows only muscovite features, sample no. 35 shows only calcite features while sample no. 9 , and 57 showed both muscovite and calcite features.

Table 5 Classifications of split B2 samples, where $\mathrm{P}, \mathrm{M}$ and $\mathrm{W}$ indicate product, middling and waste, respectively. $\mathrm{M} *$ and $\mathrm{M}$-indicates middling with more product or waste pixels respectively.

Sample ID

Cu content (BPXRF)\%

Cu content (PXRF)\%

Classification

BPXRF

PXRF

$\operatorname{NIR}(1)$

NIR(2)

1.30

2.31

3.20

1.40

0.77

1.33

0.14

1.51

2.82

8.66

8.91

1.85

0.18

4.40

4.06

5.78

1.87

1.77

0.71

2.93

1.13

2.71

10.95

8.91

0.85

0.03

\begin{tabular}{|l|}
\hline P \\
\hline P \\
\hline P \\
\hline P \\
\hline P \\
\hline W \\
\hline P \\
\hline P \\
\hline P \\
\hline P \\
\hline P \\
\hline W \\
\hline
\end{tabular}

\begin{tabular}{|c|c|c|}
\hline$P$ & $P$ & $P$ \\
\hline$P$ & $P$ & $P$ \\
\hline$P$ & $P$ & $P$ \\
\hline$P$ & $\mathrm{M}^{*}(\mathrm{P})$ & $P$ \\
\hline$P$ & $P$ & $P$ \\
\hline$P$ & $P$ & $P$ \\
\hline$P$ & $P$ & $P$ \\
\hline$P$ & $P$ & $P$ \\
\hline$P$ & w & $w$ \\
\hline$P$ & $P$ & $P$ \\
\hline$P$ & $P$ & $P$ \\
\hline$P$ & $P$ & $P$ \\
\hline W & M-(W) & $M-(W)$ \\
\hline
\end{tabular}




\section{ELSEVIER_MINE_4780}

\begin{tabular}{|c|c|c|c|c|c|c|}
\hline 14 & 0.49 & 1.52 & w & $P$ & $\mathrm{M}^{*}(\mathrm{P})$ & $P$ \\
\hline 15 & 0.03 & 0.03 & w & w & $M-(W)$ & $P$ \\
\hline 16 & 0.35 & 1.31 & w & $P$ & $\mathrm{P}$ & $P$ \\
\hline 17 & 0.34 & 2.04 & w & $\mathrm{P}$ & P & $P$ \\
\hline 18 & 0.13 & 4.39 & w & $P$ & $M-(W)$ & $P$ \\
\hline 19 & 0.25 & 0.61 & w & $P$ & $\mathrm{P}$ & $P$ \\
\hline 20 & 0.18 & 0.65 & w & $P$ & $\mathrm{P}$ & $P$ \\
\hline 21 & 0.09 & 0.17 & w & w & $\mathrm{M}^{*}(\mathrm{P})$ & $P$ \\
\hline 22 & 0.08 & 0.62 & w & $\mathrm{P}$ & P & $P$ \\
\hline 23 & 0.09 & 2.58 & w & P & $\mathrm{P}$ & $P$ \\
\hline 24 & 3.30 & 0.14 & $P$ & w & $M-(W)$ & $P$ \\
\hline 25 & 0.74 & 0.33 & $P$ & w & $M-(W)$ & $M-(W)$ \\
\hline 26 & 0.45 & 0.88 & w & $P$ & $\mathrm{P}$ & $P$ \\
\hline 27 & 0.02 & 0.05 & w & w & w & w \\
\hline 28 & 0.73 & 0.30 & $P$ & w & $\mathrm{P}$ & $P$ \\
\hline 29 & 0.10 & 0.09 & w & w & w & $P$ \\
\hline 30 & 0.24 & 0.15 & w & w & P & $P$ \\
\hline 31 & 0.22 & 0.46 & w & w & $\mathrm{P}$ & $P$ \\
\hline 32 & 0.89 & 0.88 & $P$ & $P$ & $\mathrm{P}$ & $P$ \\
\hline 33 & 0.03 & 0.07 & w & w & $\mathrm{P}$ & $P$ \\
\hline 34 & 0.21 & 0.21 & w & w & P & $P$ \\
\hline 35 & 0.89 & 2.13 & $P$ & P & w & w \\
\hline 36 & 0.06 & 0.14 & w & w & $\mathrm{P}$ & $P$ \\
\hline 37 & 0.12 & 1.42 & w & $P$ & P & $P$ \\
\hline 38 & 0.22 & 0.69 & w & $P$ & $P$ & $P$ \\
\hline 39 & 0.14 & 0.16 & w & w & $M^{*}(\mathrm{P})$ & $\mathrm{M}^{*}(\mathrm{P})$ \\
\hline 40 & 0.44 & 1.78 & w & $\mathrm{P}$ & P & $P$ \\
\hline 41 & 0.05 & 0.03 & w & w & $\mathrm{P}$ & $P$ \\
\hline 42 & 0.11 & 0.25 & w & w & w & w \\
\hline 43 & 0.13 & 1.31 & w & $\mathrm{P}$ & $\mathrm{M}^{*}(\mathrm{P})$ & $\mathrm{M}^{*}(\mathrm{P})$ \\
\hline 44 & 0.21 & 5.88 & w & $P$ & $P$ & $P$ \\
\hline 45 & 0.11 & 2.01 & w & $\mathrm{P}$ & P & $P$ \\
\hline
\end{tabular}




\section{ELSEVIER_MINE_4780}

\begin{tabular}{|c|c|c|c|c|c|c|}
\hline 46 & 0.16 & 0.11 & w & w & w & w \\
\hline 47 & 0.46 & 0.17 & w & w & w & w \\
\hline 48 & 0.36 & 0.43 & w & w & $\mathrm{M}^{*}(\mathrm{P})$ & $\mathrm{M}^{*}(\mathrm{P})$ \\
\hline 49 & 0.13 & 0.31 & w & w & $M-(W)$ & $P$ \\
\hline 50 & 2.08 & 3.87 & $P$ & $\mathrm{P}$ & $P$ & $P$ \\
\hline 51 & 1.39 & 0.67 & $P$ & $P$ & $P$ & $P$ \\
\hline 52 & 0.14 & 0.11 & w & w & w & w \\
\hline 53 & 0.09 & 0.09 & w & w & w & w \\
\hline 54 & 0.23 & 0.65 & w & $P$ & w & $P$ \\
\hline 55 & 3.03 & 3.93 & $P$ & $P$ & w & $P$ \\
\hline 56 & 0.33 & 0.17 & w & w & w & w \\
\hline 57 & 2.28 & 1.02 & $\mathrm{P}$ & $\mathrm{P}$ & w & w \\
\hline 58 & 0.07 & 0.09 & w & w & $\mathrm{P}$ & $P$ \\
\hline 59 & 0.19 & 0.18 & w & w & P & $P$ \\
\hline 60 & 4.44 & 1.97 & $P$ & $P$ & P & $P$ \\
\hline
\end{tabular}

The second strategy, NIR (2), classified only 12 samples as waste, compared to the 19 samples classified as waste by the NIR (1). Hence, product concentrate by NIR (2) consist of 48 samples.

\section{Evaluation of results}

Ore parameters for each split were calculated based on the assumption that the mass of each sample were the same.

\subsection{Splits B1}

Classification of 60 samples is presented in Table 4. Tables $6 a$ and $6 \mathrm{~b}$ present calculated parameters of splits B1.

Table 6a Comparison of discrimination results of splits B1, using copper content calculated from copper-bearing minerals Qem.

\begin{tabular}{|c|c|c|c|c|c|}
\hline & NIR (1) & NIR (2) & Qem & Func. (1) & Func. (2) \\
\hline Content of copper in feed, \% & 1.12 & 1.12 & 1.12 & 1.12 & 1.12 \\
\hline Content of copper in product, \% & 1.47 & 1.22 & 2.77 & 2.11 & 3.06 \\
\hline Content of copper in waste, \% & 0.49 & 0.38 & 0.10 & 0.97 & 0.36 \\
\hline Recovery of $\mathrm{Cu}$ in product, \% & 85 & 96 & 94 & 25 & 77 \\
\hline Yield of product, \% & 65 & 88 & 38 & 13 & 28 \\
\hline
\end{tabular}

Table 6b Comparison of discrimination results of splits B1, using copper content measured by PXRF.

$\begin{array}{lllll}\text { NIR (1) NIR (2) PXRF } & \text { Punc. (1) } & \text { Func. (2) }\end{array}$




\section{ELSEVIER_MINE_4780}

\section{Content of copper in product, \%}

Content of copper in waste, \%

Recovery of $\mathrm{Cu}$ in product, \%

Yield of product, \%
1.03

0.82

1.03

0.41

95

88

\begin{tabular}{|l|l|}
\hline & 2.42 \\
\hline 0.11 \\
93 \\
37
\end{tabular}

37

\begin{tabular}{|l|l}
\hline 42 & 1.56 \\
\hline 11 & 0.87 \\
\hline 3 & 21 \\
\hline & 13
\end{tabular}

13
2.00

0.54

59

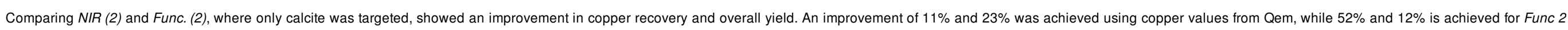

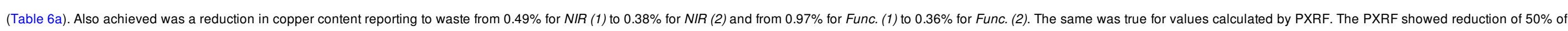
the total copper reporting to waste for NIR (1), with improvement in copper recovery of $25 \%$ (Table 6b). Similar improvement was achieved for Func. (2) against Func. (1).

\subsection{Splits B2}

Classification of splits B2 samples is presented in Table 5.The calculated parameters are presented in Tables 7a and 7b using data from PXRF and BPXRF respectively.

Table 7a Comparison of discrimination results of splits B2, using copper content measured by PXRF.

\begin{tabular}{|c|c|c|c|}
\hline & NIR (1) & NIR (2) & PXRF \\
\hline Content of copper in feed, \% & 1.55 & 1.55 & 1.55 \\
\hline Content of copper in product, \% & 1.87 & 1.79 & 2.54 \\
\hline Content of copper in waste, \% & 0.88 & 0.60 & 0.17 \\
\hline Recovery of $\mathrm{Cu}$ in product, \% & 82 & 92 & 95 \\
\hline Yield of product, \% & 68 & 80 & 58 \\
\hline
\end{tabular}

Table 7b Comparison of discrimination results of splits B2, using copper content measured on powdered samples by PXRF.

\begin{tabular}{|c|c|c|c|}
\hline & NIR (1) & NIR (2) & BPXRF \\
\hline Content of copper in feed, \% & 1.02 & 1.02 & 1.02 \\
\hline Content of copper in product, \% & 1.12 & 1.10 & 2.56 \\
\hline Content of copper in waste, \% & 0.80 & 0.68 & 0.19 \\
\hline Recovery of $\mathrm{Cu}$ in product, \% & 80 & 87 & 88 \\
\hline Yield of product, \% & 68 & 80 & 35 \\
\hline
\end{tabular}

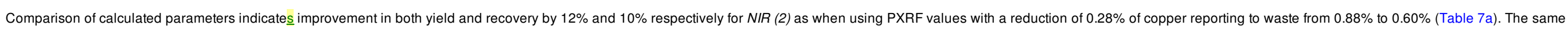
was true using values from BPXRF (Table $7 \mathrm{~b}$ ), showing an improvement of $7 \%$ in recovery and $12 \%$ in yield.

\section{Implication of NIR sorting}

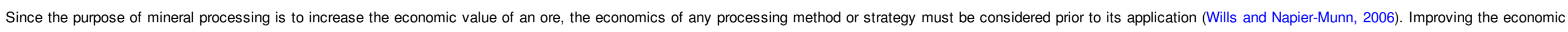

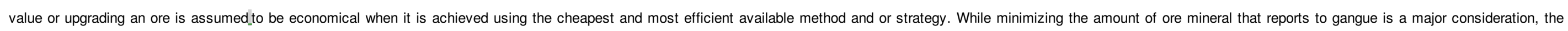
recovery is critical.

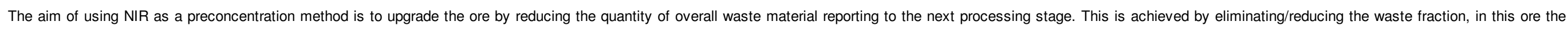

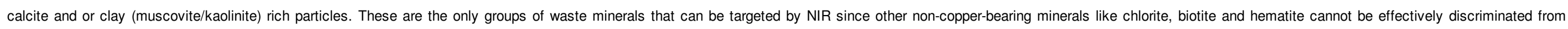




\section{ELSEVIER_MINE_4780}

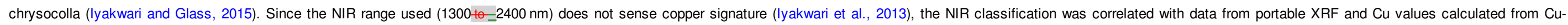

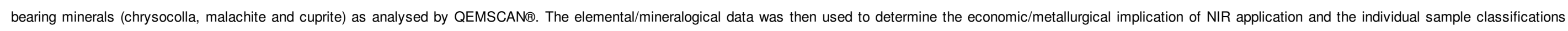
based on NIR, functional groups content as well as copper content are compared.

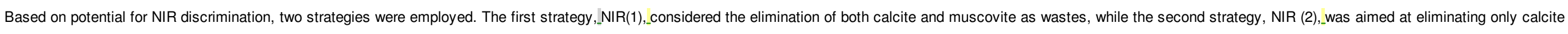

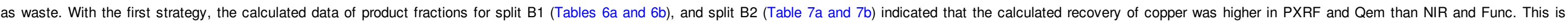

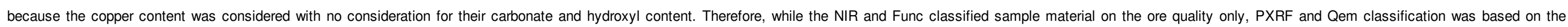
quantity of copper in a sample. Also observed from NIR (1) and Func. (1) was the high percentage of copper lost to waste, in most cases with values higher than the assumed cut-off grade (0.5\%).

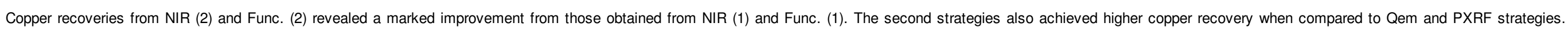

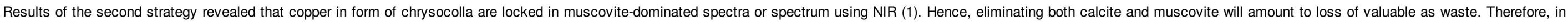

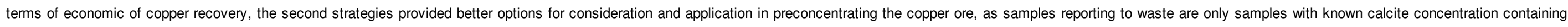
little or no chrysocolla. Therefore, although the first strategy provides fairly pure concentrate, the second strategy minimizes the loss of valuable as waste.

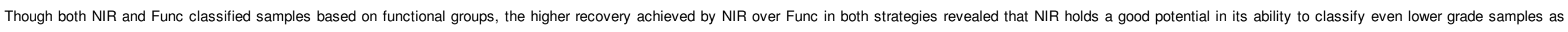
products provided those samples show no calcite and or clay minerals absorption features.

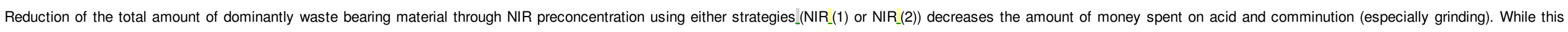
could constitute up to $50 \%$ of the total energy cost used in mineral processing operation (Wills and Napier-Munn, 2006), the total volume of the waste rejection leads to a proportional reduction in the total energy cost.

\section{Conclusions}

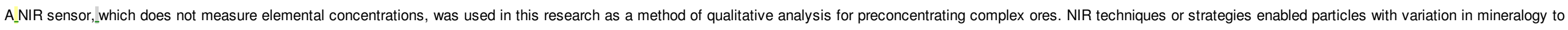

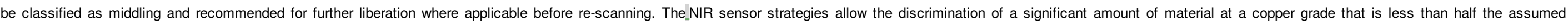
economic cut-off-grade of $0.5 \%$. This indicates that there is potential that the NIR sensor based sorting can provide economic benefits for processing ore.

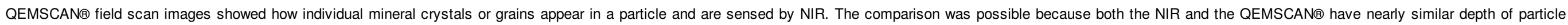

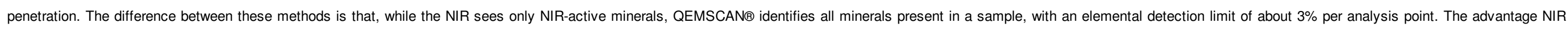
has over QEMSCAN $\circledast$ is its ability to detect NIR-active functional groups in minerals $\left(\mathrm{H}_{2} \mathrm{O},-\mathrm{OH}\right.$ and $\left.\mathrm{CO}_{3}^{2-}\right)$ quickly without rigorous sample preparation.

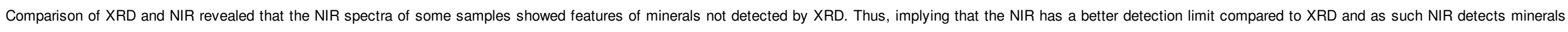

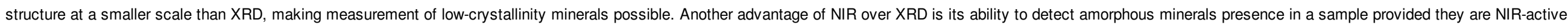

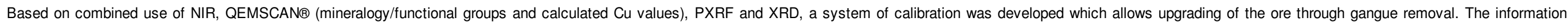

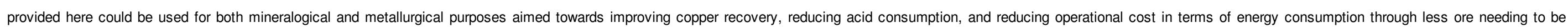
processed.

\section{Uneited referenees}

Andresen al. (2009), Glarketal. (1990), Clark (1999), Halbieh (1934).

\section{Acknowledgments}

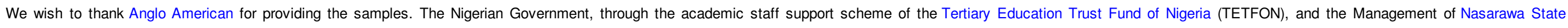
University, Keffi, are acknowledged for providing financial support for this research.

\section{References}




\section{ELSEVIER_MINE_4780}

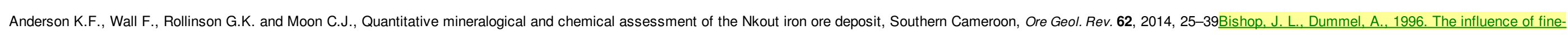

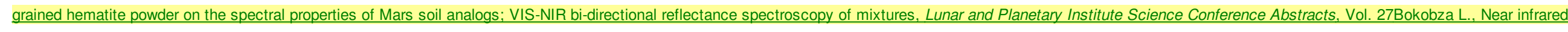
spectroscopy. J. Near Infrared Spectrosc., 6. 1998, 3-7.

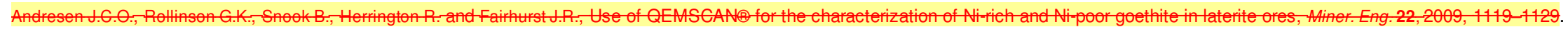

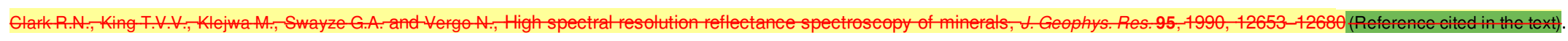

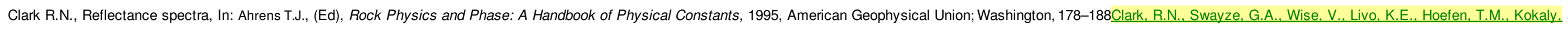
R.F., Sutley, S.J., 2007. USGS digital spectral library splib06a: U.S. Geological Survey, Data Series 231.

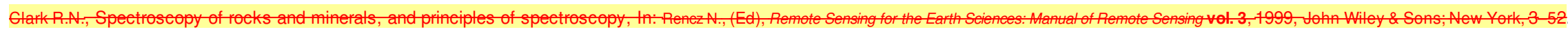

Coates J., Interpretation of infrared spectra, a practical approach, In: Meyers R.A., (Ed), Encyclopaedia of Analytical Chemistry, 2000, Wiley \& Sons Ltd.; Chichester, $10815-10837$.

Curtis B., Developing automated copper ore processing using NIR analysis and XRD, Adv. Mater. Processes 170 (2), 2012, 24-26.

Dalm M., Buxton M.W., van Ruitenbeek F.J. and Voncken J.H., Application of near-infrared spectroscopy to sensor based sorting of a porphyry copper ore, Miner. Eng. 58, $2014,7-16$.

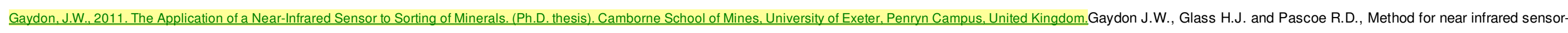
based sorting of a copper ore, J. Near Infrared Spectrosc. 17, 2009, 177-194.

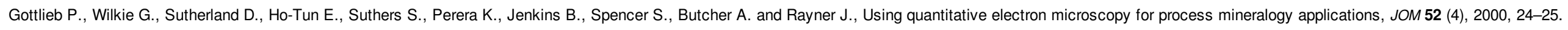

Gupta K.C., Chemical Metallurgy: Principles and Practice, 2003, WILEY-VCH Verlag GmbH \& Co; Weinheim. ISBN: 3-527-30376-6.

Habashi F., Handbook of extractive metallurgy. Vol. II: primary metals, secondary metals, Light Metals 1997, WILEY-VCH; Weinheim, ISBN: 3-527-28792-2.

Halbieh W., Über die Anwendungsmögliehkeiteneiniger Netzmittel in der Flotation, 1934, KondradTriltsch; Würzburg.

Hunt G.R., Spectral signatures of particulate minerals in the visible and near-infrared, Geophysics 42 (3), 1977, 501-513.

lyakwari S., Glass H.J. and Kowalczuk P.B., Potential for near infrared sensor-based sorting of hydrothermally-formed minerals, J. Near Infrared Spectrosc. 21 (3), $2013,223-229$.

lyakwari S. and Glass H.J., Influence of mineral particle size and choice of suitable parameters for ore sorting using near infrared sensors, Miner. Eng. 69, 2014, 102-106.

lyakwari S. and Glass H.J., Mineral preconcentration using near infrared sensor-based sorting, Physicochem. Probl. Miner. Process. 51 (2), $2015,661-674$.

Li L., Wu Q., Li S. and Wu O., Study of the infrared spectral features of an epoxy curing mechanism, Appl. Spectrosc. 62 (10), 2008, 1129-1136.

Pasquini C., Near infrared spectroscopy: fundamentals, practical aspects and analytical applications, J. Braz. Chem. Soc. 14 (2), 2003, 198-219.

Pirrie D. and Rollinson G.K., Unlocking the applications of automated mineral analysis, Geol. Today 27 (6), 2011, 235-244.

Povarennykh A.S., The use of infrared spectra for the determination of minerals, Am. Mineral. 63, 1978, 956-959.

Reich G., Near-infrared spectroscopy and imaging: basic principles and pharmaceutical application, Adv. Drug Deliv. Rev. 57, 2005, 1109-1143.

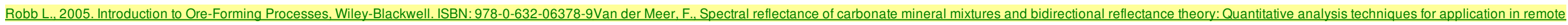

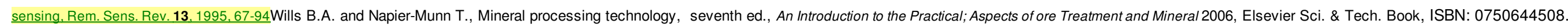

Graphical abstract 


\section{ELSEVIER_MINE_4780}

Copper samples preconcentration strategy targeted at eliminating only calcite-bearing samples as waste.

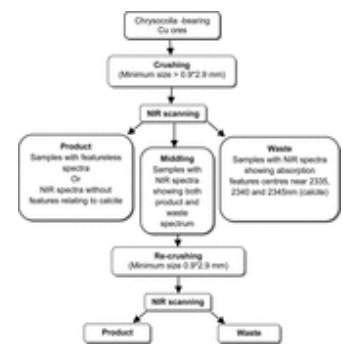

Highlights

- Combination of near infrared and QEMSCAN technologies to aid ore recovery

- Near infrared discrimination correlates well with mineralogical and chemical classifications.

- Preconcentration of copper ore by near infrared sensor-based sorting is proposed.

\section{Queries and Answers}

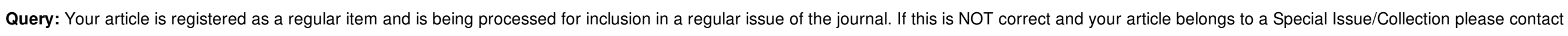
n.rajaj@elsevier.com immediately prior to returning your corrections.

Answer: yes it is a regular item

Query: Please confirm that given name(s) and surname(s) have been identified correctly.

Answer: they are all correct

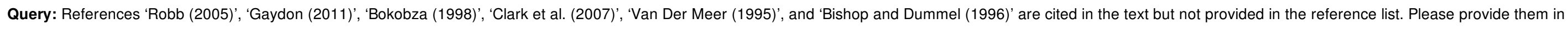
the reference list or delete these citations from the text.

Answer: References inserted

Query: Please check the hierarchy of the section headings.

Answer: checked and are all correct

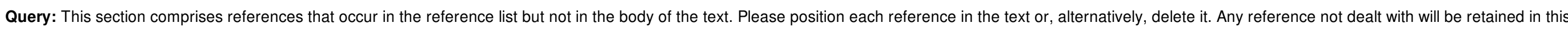
section.

Answer: All deleted 